\title{
Linear and Branched Phospha[n]triangulanes
}

\author{
J. Chris Slootweg, ${ }^{[a]}$ Frans J. J. de Kanter, ${ }^{[a]}$ Marius Schakel, ${ }^{[a]}$ Martin Lutz, ${ }^{[b]}$ \\ Anthony L. Spek, ${ }^{[b]}$ Sergei I. Kozhushkov, ${ }^{[c]}$ Armin de Meijere, ${ }^{[c]}$ and \\ Koop Lammertsma*[a]
}

\author{
Dedicated to Professor Paul von Ragué Schleyer on the occasion of his 75th birthday
}

\begin{abstract}
Novel, highly stable, linear and branched mono- and diphospha $[n]$ triangulanes were synthesized in high yields by the $\mathrm{CuCl}$-catalyzed phosphinidene addition to spirocyclopropanated methylenecyclopropanes and bicyclopropylidenes. The effect of spirofusion on the electronic properties of these esthetically attractive phosphacycles is apparent from X-ray single crystal structure analyses, which reveals a tightening of the phosphirane ring on
\end{abstract}

additional spirocyclopropanation, and from the NMR features that show deshielded chemical shifts for the ringphosphorus and -carbon atoms. Steric factors play a role in the addition reaction when the substrate alkene carries a second sphere of spirocyclopropane

Keywords: copper $\cdot$ cycloaddition heterocycles $\cdot$ phosphorus $\cdot$ triangulanes rings and causes the formation of 2phosphabicyclo[3.2.0]heptenes in small amounts. These by-products most probably result from addition of the $\left[\mathrm{PhP}(\mathrm{Cl}) \mathrm{W}(\mathrm{CO})_{5}\right]-\mathrm{Cu}-\mathrm{L}(\mathrm{L}=$ alkene or solvent) reagent to the spirocyclopropanated bicyclopropylidene to give an intermediate $\sigma$-complex, which subsequently, facilitated by steric factors, undergoes a cyclopropylcarbinyl to cyclobutyl ring expansion followed by a $[1,3]$-sigmatropic shift.

\section{Introduction}

The inherently strained cyclopropane derivatives have unique electronic and chemical properties due to their small valence angles and bent $\mathrm{C}-\mathrm{C}$ bonds. ${ }^{[1]}$ Embedding the threemembered ring into oligocycles by annelation or spirofusion with additional small rings augments the total strain to exceed that of the sum of the separate rings. This is well established not only for the ring-annelated bicyclic (1) and tri-

[a] J. C. Slootweg, Dr. F. J. J. de Kanter, Dr. M. Schakel, Prof. Dr. K. Lammertsma

Department of Organic and Inorganic Chemistry

Faculty of Sciences, Vrije Universiteit, De Boelelaan 1083

$1081 \mathrm{HV}$, Amsterdam (The Netherlands)

Fax: $(+31) 20-598-7488$

E-mail: lammert@chem.vu.nl

[b] Dr. M. Lutz, Prof. Dr. A. L. Spek

Bijvoet Center for Biomolecular Research Crystal and Structural Chemistry, Utrecht University Padualaan 8, 3584 CH, Utrecht (The Netherlands)

[c] Dr. S. I. Kozhushkov, Prof. A. de Meijere Institut für Organische Chemie der Georg-August-Universität Göttingen Tammannstrasse 2, 37077 Göttingen (Germany) cyclic propellane skeletons (2), but also for the spirofused linear and branched so-called $[n]$ triangulanes $(\mathbf{3}) \cdot{ }^{[2]}$ The current record of a branched [15]triangulane ${ }^{[3]}$ and linear [9]triangulane ${ }^{[3]}$ exemplifies the accessibility of such extended arrays of spirofused cyclopropanes that are surprisingly stable in spite of their high overall strain.

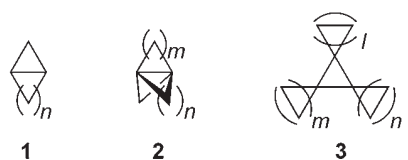

By contrast to the large number of purely carbon-based triangulanes, far fewer spirocyclic compounds are known that contain a heteroatom, because of the higher reactivity of three-membered heterocycles. ${ }^{[2]}$ Compounds such as 1 phosphaspiro[2,n]alkanes $\mathbf{4 a - e},^{[4]}$ the 1-phosphadispiroalkanes 5a-d ${ }^{[5,6]}$ and 1,4-diphosphaspiropentane $\mathbf{6}^{[7]}$ with a phosphorus atom, that is, a phosphirane ring, ${ }^{[8]}$ became accessible by the $[1+2]$ cycloaddition of the in situ generated carbene-like electrophilic phosphinidene complexes $\mathrm{R}-\mathrm{P}=$ $\mathrm{W}(\mathrm{CO})_{5}$ to the respective alkenes. ${ }^{[9,10]}$ 


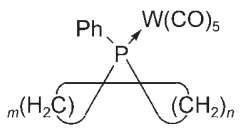

4a-e: $m=0 ; n=0,2-5$ 5a-d: $m=2 ; n=2-5$
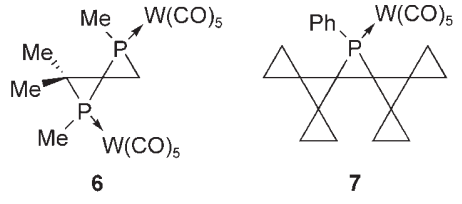

Recently, we described the synthesis of the first hetero[7]triangulane $\mathbf{7}$ and showed that even the demetallated compound is stable at $150^{\circ} \mathrm{C} \cdot{ }^{[11]} \mathrm{In}$ the present report we expand on these earlier studies and describe a series of novel stable phospha $[n]$ triangulanes containing one or two phosphorus atoms.

\section{Results and Discussion}

The syntheses of phospha $[n]$ triangulanes $(n=3-5)$ was achieved by adding $\mathrm{Ph}-\mathrm{P}=\mathrm{W}(\mathrm{CO})_{5}$ to terminal and non-terminal double bonds of spirocyclopropanated methylenecyclopropanes and bicyclopropylidenes, respectively. The influence of the $\mathrm{CuCl}$ catalyst on the addition reaction will also be addressed.

Spirocyclopropanated methylenecyclopropanes, that is, terminal alkenes: Reaction of $\mathrm{Ph}-\mathrm{P}=\mathrm{W}(\mathrm{CO})_{5}$, generated in situ by the $\mathrm{CuCl}$-catalyzed cycloreversion ${ }^{[12]}$ of 7-phosphanorbornadiene complex $\mathbf{8},{ }^{[9,13]}$ with methylenespiropentane $(\mathbf{9})$ in toluene at $55^{\circ} \mathrm{C}(1 \mathrm{~h})$ gave in $78 \%$ yield only the crystalline terminal phospha[3]triangulane complex 12 (Scheme 1) in a
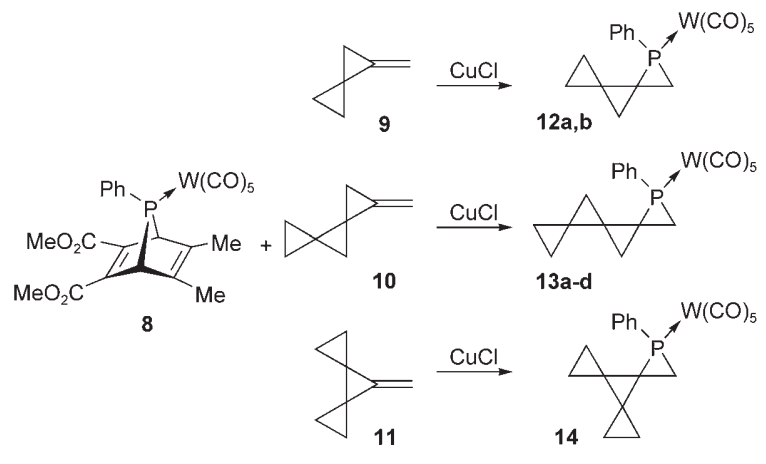
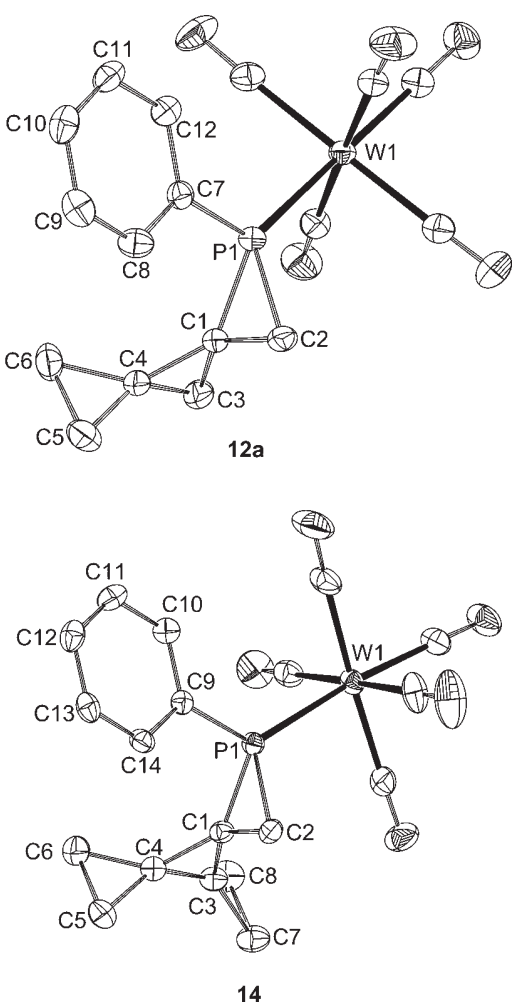

Figure 1. Displacement ellipsoid plot of $\mathbf{1 2 a}$ (one of the two crystallographically independent molecules is shown) and $\mathbf{1 4}$ in the crystal with ellipsoids set at the $50 \%$ probability level. Hydrogen atoms are omitted for clarity. Selected bond lengths $[\AA]$ and angles $\left[{ }^{\circ}\right]$; the values for the second molecule are in square brackets. 12a: C4-C5 1.486(4) [1.475(4)], C4-C6 1.478(4) [1.475(4)], C5-C6 1.528(4) [1.515(4)]; C1-P1-C2 48.41(11) [48.25(12)], C3-C1-C4 59.86(18) [60.33(18)], C5-C4-C6 62.1(2) [61.8(2)]. 14: C3-C7 1.478(3), C3-C8 1.475(3), C4-C5 1.481(3), C4-C6 1.482(3), C5-C6 1.537(4), C7-C8 1.522(4); C1-P1-C2 48.36(10), C3-C1C4 59.01(14), C5-C4-C6 62.50(17), C7-C3-C8 62.03(16).

phospha[2]triangulane $\mathbf{4 b}^{[4]}$ (Table 1). Extending the number of cyclopropane rings to three, as in linear $\mathbf{1 0}$ or branched methylenetriangulane 11, gave the corresponding linear and branched phospha[4]triangulanes $\mathbf{1 3}$ and $\mathbf{1 4}$ (Scheme 1). In the case of $\mathbf{1 4}$, only one isomer can be formed $(88 \%)$ of which the X-ray crystal structure is shown in Figure 1, but four diastereomers are feasible for linear $\mathbf{1 3}$ (94\%), which depends on the syn,anti relationship of the phosphirane and terminal spirocyclopropane rings as well as

Scheme 1.

$5: 4$ ratio of the anti (a) and syn isomer (b). Figure 1 shows the $\mathrm{X}$-ray crystal structure for the less congested anti isomer $\mathbf{1 2 a}$ that has the $\mathrm{P}-\mathrm{W}(\mathrm{CO})_{5}$ group anti to the terminal cyclopropane ring. The bond lengths of the phosphirane ring compare well with the values of the $\mathrm{W}(\mathrm{CO})_{5}$-complexed smaller
Table 1. Selected X-ray crystallographic bond lengths for the phosphirane ring of terminal phospha[ $n]$ triangulanes.

\begin{tabular}{|c|c|c|c|c|c|c|c|c|c|}
\hline \multirow[b]{2}{*}{$\mathrm{P} 1-\mathrm{C} 1$} & \multirow{2}{*}{$\frac{\mathbf{4} \mathbf{b}^{[4]}}{1.794(6)}$} & \multicolumn{2}{|c|}{$12 \mathbf{a}^{[\mathrm{a}]}$} & \multirow{2}{*}{$\frac{14}{1.801(2)}$} & \multicolumn{2}{|c|}{$16 b^{[a]}$} & \multicolumn{2}{|c|}{$17 a$} & \multirow{2}{*}{$\begin{array}{r}\mathrm{av}^{*} \\
1.796\end{array}$} \\
\hline & & $1.799(2)$ & $1.802(2)$ & & $1.796(8)$ & $1.792(8)$ & $1.792(6)$ & $1.792(6)$ & \\
\hline $\mathrm{P} 1-\mathrm{C} 2$ & $1.855(7)$ & $1.846(3)$ & $1.846(3)$ & $1.836(2)$ & $1.860(8)$ & $1.850(8)$ & $1.841(6)$ & $1.846(6)$ & 1.846 \\
\hline $\mathrm{C} 1-\mathrm{C} 2$ & $1.508(9)$ & $1.495(4)$ & $1.492(4)$ & $1.490(3)$ & $1.499(11)$ & $1.501(11)$ & $1.503(9)$ & $1.507(9)$ & 1.498 \\
\hline $\mathrm{C} 1-\mathrm{C} 3$ & $1.470(1)$ & $1.504(4)$ & $1.504(4)$ & $1.486(3)$ & $1.505(11)$ & $1.494(11)$ & $1.505(8)$ & $1.505(8)$ & $1.503^{[\mathrm{b}]}$ \\
\hline $\mathrm{C} 1-\mathrm{C} 4$ & $1.475(10)$ & $1.462(3)$ & $1.467(3)$ & $1.482(3)$ & $1.468(11)$ & $1.479(11)$ & $1.469(9)$ & $1.461(8)$ & $1.468^{[\mathrm{b}]}$ \\
\hline $\mathrm{C} 3-\mathrm{C} 4$ & $1.515(10)$ & $1.480(4)$ & $1.493(4)$ & $1.461(3)$ & $1.494(12)$ & $1.497(11)$ & $1.499(9)$ & $1.497(9)$ & $1.493^{[\mathrm{b}]}$ \\
\hline $\mathrm{P} 1-\mathrm{W} 1$ & $2.500(2)$ & $2.5107(7)$ & $2.4980(7)$ & $2.5063(5)$ & $2.497(2)$ & $2.497(2)$ & $2.4822(16)$ & $2.4733(16)$ & 2.495 \\
\hline
\end{tabular}

[a] Both crystallographically independent molecules are given. * Average of 12a, 14, 16b, and 17a. [b] Excluding 14 because of its symmetrical substituent pattern. 
that of the P-substituents. The less congested two major isomers of the four observed by ${ }^{31} \mathrm{P}$ NMR spectroscopy (ratio 50:36:10:4) were fully characterized (see Experimental Section).

Diphospha[4]triangulanes are accessible by using racemic 1,4-dimethylenespiropentane $r a c-\mathbf{1 5}$ as diene. Reaction of the phosphinidene precursor $\mathbf{8}(\mathrm{CuCl} \text {-catalyzed })^{[12]}$ with only one double bond, with an excess of $\mathbf{1 5}$ in toluene at $55^{\circ} \mathrm{C}$ $(2 \mathrm{~h})$, afforded 5-methylene-1-phosphadispiro[2.0.2.1]heptane 16 (Scheme 2) in $83 \%$ yield as a mixture of four diastereomers $\left({ }^{31} \mathrm{P}\right.$ NMR: ratio $\left.39: 31: 18: 12\right)$ of which three could be fully characterized. The structure of the major anti isomer $16 \mathbf{b}$, separated by column chromatography, was ascertained by an X-ray crystal structure determination (Figure 2). The presence of the double bond (1.289(14) $\AA$ ) is also evident from the ${ }^{1} \mathrm{H}$ NMR resonances at $\delta=5.36\left({ }^{4} J\right.$ $(\mathrm{H}, \mathrm{H})=2.4 \mathrm{~Hz})$ and $5.39 \quad\left({ }^{4} J(\mathrm{H}, \mathrm{H})=1.8 \mathrm{~Hz}\right)$ and the ${ }^{13} \mathrm{C}$ NMR resonances at $\delta=101.6\left(\mathrm{H}_{2} C=\right)$ and $133.7(=C)$.

Reaction of the remaining double bond in anti-16b with an excess of $8\left(55^{\circ} \mathrm{C}, \mathrm{CuCl}, 3 \mathrm{~h}\right)$ resulted in the smooth formation of the novel linear diphospha[4]triangulane complex

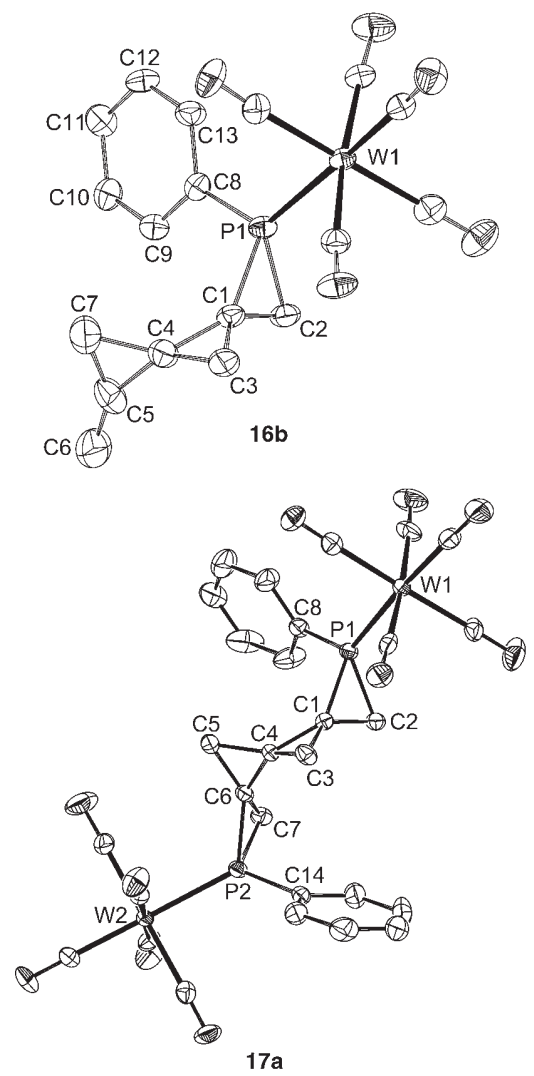

Figure 2. Displacement ellipsoid plot of $\mathbf{1 6 b}$ (one of the two crystallographically independent molecules is shown) and $\mathbf{1 7} \mathbf{a}$ in the crystal with ellipsoids set at the $50 \%$ probability level. Hydrogen atoms are omitted for clarity. Selected bond lengths $[\AA]$ and angles $\left[{ }^{\circ}\right]$; the values for the second molecule are in square brackets 16b: C4-C5 1.422(12) [1.423(12)], C4-C7 1.500(13) [1.503(13)], C5-C6 1.289(14) [1.289(14], C5-C7 1.456(14) [1.475(15)]; C1-P1-C2 48.4(4) [48.6(4)], C3-C1-C4 60.3(5) [60.5(5)], C5-C4-C7 59.7(6) [60.5(7)]. 17a: C1-P1-C2 48.9(3), C6P2-C7 48.9(3), C3-C1-C4 60.5(4), C4-C5-C6 58.2(4), C4-C6-C5 60.6(4).
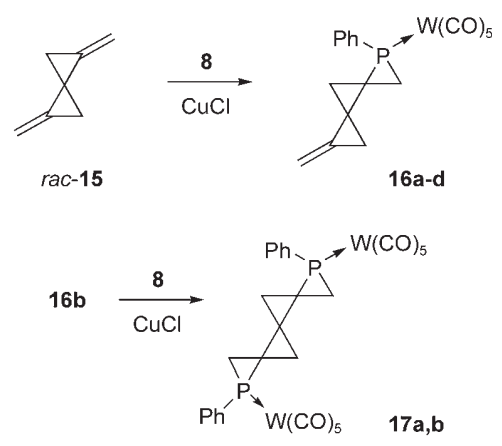

Scheme 2.

$\mathbf{1 7}$ in $78 \%$ yield in a 10:7 ratio of the anti,anti and anti,syn isomers (Scheme 2). anti,anti-isomer $\mathbf{1 7} \mathbf{a}$ is easily recognized by its ${ }^{31} \mathrm{P}$ NMR singlet $(\delta-147.9)$ and anti,syn-isomer 17b by its set of doublets $\left(\delta-150.4,-150.5 ;{ }^{4} J(\mathrm{P}, \mathrm{P})=4.5 \mathrm{~Hz}\right)$. Interestingly, the ${ }^{13} \mathrm{C}$ NMR spectrum of $\mathbf{1 7}$ a displays an $\mathrm{A}_{2} \mathrm{X}$ system for the central spiro-carbon atom at $\delta^{13} \mathrm{C}=25.4\left({ }^{2} J\right.$ $(\mathrm{C}, \mathrm{P})=5.5 \mathrm{~Hz})$. An X-ray crystal structure confirmed the assignment of this isomer (Figure 2).

Selected structural parameters of four of the novel (di)phospha $[n]$ triangulane complexes are given in Table 1 . The two different $\mathrm{P}-\mathrm{C}$ bond lengths (av 1.796 and $1.846 \AA$ ) of their terminal phosphirane rings are similar to the values of the $\mathrm{W}(\mathrm{CO})_{5}$-complexed smaller phospha[2]triangulane $\mathbf{4} \mathbf{b}^{[4]}$ with the distal bonds (remote to the spiro-carbon) being longer than the proximal ones (connected to the spirocarbon). This effect of spirofusion is similar but more pronounced than in the all-carbon triangulanes, ${ }^{[3,14]}$ and likewise is due to rehybridization of the strained spiro-carbon, resulting in less s-character and thus elongation of the distal bonds. ${ }^{[2]}$ The $\mathrm{PhPW}(\mathrm{CO})_{5}$ group also affects the $\mathrm{C} 1$-spirofused cyclopropane ring, which also has proximal $\mathrm{C}-\mathrm{C}$ bonds of different length (av 1.503 and $1.468 \AA$ ) and a $1.493 \AA$ (av) distal bond. The $\mathrm{C} 1-\mathrm{C} 2$ bonds (av $1.498 \AA$ ) of all the structures are of similar length as reported for the parent 1-phenylphosphirane $\mathbf{4 a}{ }^{[4]}$

The NMR characteristics of the phospha[n]triangulanes also reflect that their $\mathrm{W}(\mathrm{CO})_{5}$-complexed phosphirane rings have similar electronic properties (Table 2). The phosphorus chemical shifts are in the narrow range of $\delta^{31} \mathrm{P}-144$ to $-155 \mathrm{ppm}$ with ${ }^{1} J(\mathrm{P}, \mathrm{W})$ coupling constants of $252-261 \mathrm{~Hz}$. The less congested anti isomers are always formed predominantly ${ }^{[15]}$ and have the more shielded ${ }^{31} \mathrm{P}$ NMR resonances ${ }^{[16]}$ that are similar to the $\delta-154.8 \mathrm{ppm}$ reported for the parent 1-phenylphospha[2]triangulane complex $\mathbf{4 b} .{ }^{[4]}$ The ${ }^{13} \mathrm{C}$ resonance of the phosphirane spiro-carbon $\mathrm{C} 1$ and carbon $\mathrm{C} 2$ are at $\delta$ 25-30 (4b 31.2) and $\delta 15-18$ ppm (4b 19.3), respectively. In addition, its two hydrogen atoms resonate at about $\delta^{1} \mathrm{H} 1.7$ and $2.0 \mathrm{ppm}$ with ${ }^{2} J(\mathrm{H}, \mathrm{P})$ coupling constants of $<1$ and $7.1 \mathrm{~Hz}$, respectively, with the larger one for the more deshielded proton resonance positioned anti to the $\mathrm{P}$ $\mathrm{W}(\mathrm{CO})_{5}$ group.

Spirocyclopropanated bicyclopropylidenes, that is, non-terminal alkenes: Alkenes with four alkyl substituents are 
Table 2. Selected NMR parameters for the phosphirane ring of terminal phospha $[n]$ triangulanes.

\begin{tabular}{|c|c|c|c|c|c|c|c|c|c|c|c|}
\hline \multirow[b]{2}{*}{${ }^{31} \mathrm{P}$} & \multirow{2}{*}{$\begin{array}{c}\text { 12a } \\
-146.4\end{array}$} & \multirow{2}{*}{$\begin{array}{c}\mathbf{1 2 b} \\
-152.3\end{array}$} & \multirow{2}{*}{$\begin{array}{c}\mathbf{1 3 b} \\
-152.2\end{array}$} & \multirow{2}{*}{$\begin{array}{c}\text { 13d } \\
-154.9\end{array}$} & \multirow{2}{*}{$\frac{\mathbf{1 4}}{-145.2}$} & \multirow{2}{*}{$\begin{array}{c}\mathbf{1 6 b} \\
-144.0\end{array}$} & \multirow{2}{*}{$\begin{array}{c}\mathbf{1 6 c} \\
-148.3\end{array}$} & \multirow{2}{*}{$\begin{array}{c}\mathbf{1 6 d} \\
-148.5\end{array}$} & \multirow{2}{*}{$\begin{array}{c}\mathbf{1 7} \mathbf{a} \\
-147.9\end{array}$} & \multicolumn{2}{|c|}{ 17b } \\
\hline & & & & & & & & & & -150.4 & -150.5 \\
\hline${ }^{1} J(\mathrm{P}, \mathrm{W})$ & 257.2 & 254.1 & 258.1 & 255.5 & 252.1 & 258.2 & 257.2 & 256.2 & 261.2 & 260.2 & 258.2 \\
\hline${ }^{13} \mathrm{C}(\mathrm{C} 1)$ & 26.5 & 25.7 & 26.7 & 25.6 & 29.6 & 29.0 & 29.1 & 28.3 & 26.3 & 25.6 & 26.1 \\
\hline${ }^{1} J(\mathrm{C} 1, \mathrm{P})$ & 6.1 & 7.1 & 6.7 & 8.6 & 10.1 & 7.7 & 7.5 & 9.3 & 8.8 & 9.8 & 5.5 \\
\hline${ }^{13} \mathrm{C}\left(\mathrm{C} 2 \mathrm{H}_{2}\right)$ & 17.0 & 17.4 & 16.7 & 16.4 & 15.5 & 17.8 & 17.5 & 17.9 & 15.3 & 15.5 & 16.0 \\
\hline${ }^{1} J(\mathrm{C} 2, \mathrm{P})$ & $\sim 6$ & 6.1 & 6.3 & 6.4 & 5.7 & 5.8 & 6.1 & 6.0 & 6.3 & 6.1 & 6.3 \\
\hline${ }^{1} \mathrm{H}\left(\mathrm{C} 2 \mathrm{H}_{2}\right)$ & 1.79 & $\sim 1.6$ & $\sim 1.7$ & $\sim 1.7$ & 1.62 & 1.83 & 1.70 & 1.65 & 1.59 & 1.75 & $\sim 1.8$ \\
\hline \multirow[t]{2}{*}{${ }^{2} J(\mathrm{H}, \mathrm{P})$} & - & - & - & - & 0.9 & 1.2 & 0.8 & - & $\sim 7$ & - & unresolved \\
\hline & 7.1 & 7.1 & 7.1 & 7.1 & 7.0 & 7.4 & 7.0 & $\sim 7$ & 7.1 & 6.9 & unresolved \\
\hline
\end{tabular}

more reactive toward electrophiles than terminal alkenes, because of their higher nucleophilicity, yet steric factors can hamper the access to the double bond. This is known for the addition of carbenes (e.g. $\mathrm{MeCCl})^{[17]}$ but is less clear for electrophilic phosphinidenes. Reactions of transient $\mathrm{R}-\mathrm{P}=$ $\mathrm{W}(\mathrm{CO})_{5}$ invariably have first-order kinetics for the uncatalyzed cheletropic elimination from phosphanorbornadiene precursor $\mathbf{8}^{\left[{ }^{[18]}\right.}$ Competition reactions showed no discrimination between simple substituted alkenes, ${ }^{[15]}$ but the formation of the phosphaspiroalkanes $\mathbf{4 b}-\mathbf{e}$ from methylenecycloalkanes $\mathrm{H}_{2} \mathrm{C}=\mathrm{C}\left(\mathrm{CH}_{2}\right)_{n}$ occurs at different rates $(n=4$ $>3>5>2$ ) in the presence of $\mathrm{CuCl}^{[4]}$ The catalyst lowers the decomposition temperature for $\mathbf{8}$ from about 110 to $55^{\circ} \mathrm{C}$ and can alter the kinetics of the alkene addition reaction. ${ }^{[19]}$ Recently, a computational study addressed the role of the $\mathrm{CuCl}$ catalyst, showing a) that the alkene may complex to it and b) that the active reagent is likely $\left[\mathrm{RP}(\mathrm{Cl}) \mathrm{W}(\mathrm{CO})_{5}\right]-\mathrm{Cu}-\mathrm{L}$, with $\mathrm{L}$ being the alkene or solvent. ${ }^{[20]}$ In this section, the reactivity of spirocyclopropanated bicyclopropylidenes is first compared with that of the methylenecyclopropanes, while the influence of the $\mathrm{CuCl}$ catalyst is addressed in the next paragraph.

Bicyclopropylidene ${ }^{[21]}$ is more reactive toward the $\mathrm{CuCl}-$ generated phosphinidene complex than both methylenecyclopropane $^{[4]}$ and 2,3-dimethyl-2-buten $e^{[15]}$ and was shown to give the phospha[3]triangulane complex 5a. ${ }^{[5]}$ Adding a second sphere of spirocyclopropane rings gives the more electron-rich alkene $\mathbf{1 8},{ }^{[3]}$ that still allows the formation of a phosphirane, yielding phospha[7]triangulane complex $\mathbf{7}^{[11]}$ as confirmed by an X-ray crystal structure determination (Figure 3). It was established that the yield of 7 decreases from 88 to $42 \%$ in the presence of $\mathrm{CuCl}$ with simultaneous formation of other products, like $19(6 \%),{ }^{[11]}$ which may illustrate the influence of steric factors (Scheme 3). Therefore, the influence of the spirocyclopropane rings on the addition of the $\mathrm{CuCl}$-generated phosphinidene complex to mono- and dispirocyclopropanated bicyclopropylidenes was examined (Scheme 3).

Reaction of $\mathbf{8}$ with cyclopropylidenespiropentane (20) in toluene at $55^{\circ} \mathrm{C}$ in the presence of $\mathrm{CuCl}$ for one hour gave only the phospha[4]triangulane complex 21 (78\%) in a $4: 3$ ratio of the fully characterized anti (a) and syn diastereomer (b). The structure of the less congested anti isomer 21 a was ascertained by an X-ray crystal structure determination
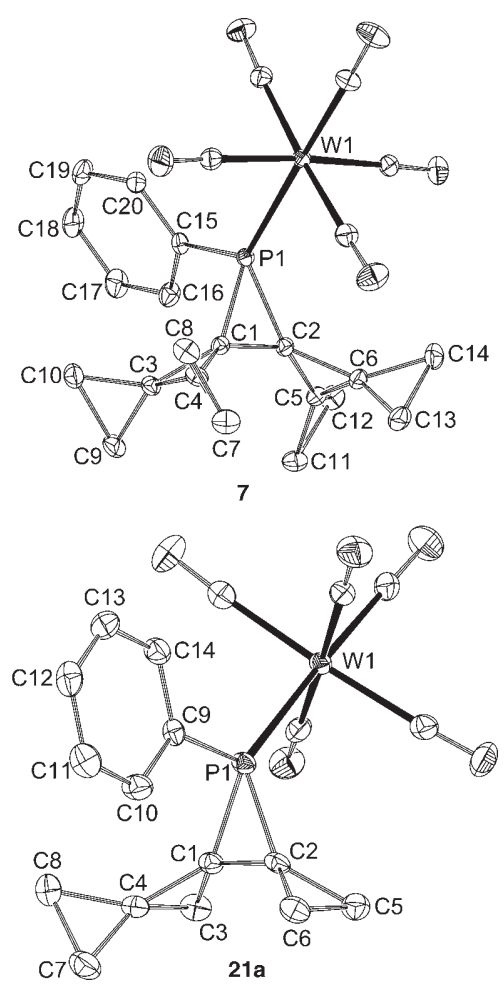

Figure 3. Displacement ellipsoid plot of $\mathbf{7}$ and $\mathbf{2 1}$ a with ellipsoids set at the $50 \%$ probability level. Hydrogen atoms are omitted for clarity. Selected bond lengths $[\AA]$ and angles $\left[{ }^{\circ}\right]$ 7: C3-C9 1.478(3), C3-C10 1.482(3), C4-C7 1.475(3), C4-C8 1.480(3), C5-C6 1.461(3), C5-C11 1.481(3), C5-C12 1.483(3), C6-C13 1.478(3), C6-C14 1.483(3), C7-C8 1.523(3), C9-C10 1.529(3), C11-C12 1.523(3), C13-C14 1.525(3); C3-C1C4 59.02(13), C9-C3-C10 62.23(14), C7-C4-C8 62.07(15), C5-C2-C6 58.64(13), C11-C5-C12 61.83(15), C13-C6-C14 62.01(15); 21 a: C4-C7 1.487(4), C4-C8 1.473(4), C7-C8 1.521(4), C3-C1-C4 59.86(17), C5-C2C6 61.10(18), C7-C4-C8 61.85(19).

(Figure 3). The bond lengths in the phosphirane ring compare well with the reported values of the smaller phospha[3]triangulane $\mathbf{5 a}^{[5]}$ (Table 3 ). The same reaction of $\mathbf{8}$ (RT, $\mathrm{CuCl}, 20 \mathrm{~h}$ ) with 7-cyclopropylidenedispiro[2.0.2.1]heptane (22) afforded the branched phospha[5]triangulane 23 (71\%) and 2-phosphabicyclo[3.2.0]heptene 24 (2\%) (Scheme 3). This reaction already takes place at room temperature, instead of the usual $55-60{ }^{\circ} \mathrm{C},{ }^{[12]}$ indicating that the bicyclopropylidene with its tetrasubstituted double bond facilitates the $\mathrm{CuCl}$-catalyzed reaction. ${ }^{[19,20]}$ It is also evident 

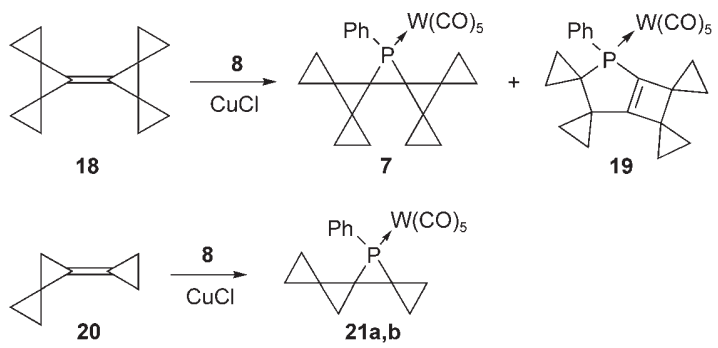

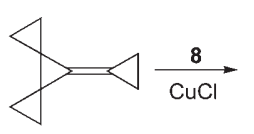

22
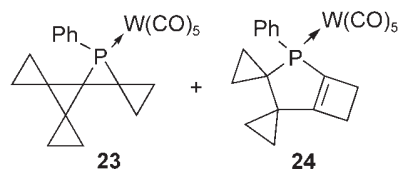

24

Scheme 3 .

common for the parent phosphiranes (e.g. major isomer anti 27 d: $\delta^{31} \mathrm{P}=-169.5$, anti $\left.26 \mathrm{a}: \delta^{31} \mathrm{P}=-127.0\right)$ (Table 4). Clearly, the $[1+2]$ cycloaddition of the phosphinidene complex to the singly substituted and less sterically congested double bond is favored over that to the more electron-rich double bond in the bicyclopropylidene moiety, suggesting that steric factors do play a role. However, reaction of the isolated anti isomer 27 d with an excess of $\mathbf{8}$ at the higher temperature of $55^{\circ} \mathrm{C}(\mathrm{CuCl}, 6.5 \mathrm{~h})$, did result in a second addition to give in $62 \%$ yield the phosphirane-substituted phospha[3]triangulane complex 28 in a 5:3 ratio of the anti,anti (a) and anti, syn isomer (b) (Scheme 4), with $12 \%$ recovery of the starting substrate $\mathbf{2 7} \mathbf{d}$. Isomer $\mathbf{2 8}$ a is easily recognized by its set of $\delta^{31} \mathrm{P}$ NMR singlets $\left(\delta-127.1,{ }^{1} J(\mathrm{P}, \mathrm{W})=258.7 \mathrm{~Hz} ;-164.0\right.$, $\left.{ }^{1} J(\mathrm{P}, \mathrm{W})=252.6 \mathrm{~Hz}\right)$ and isomer $28 \mathbf{b}$ by its set of doublets $(\delta$ -129.6, $\quad{ }^{1} J(\mathrm{P}, \mathrm{W})=259.7 \mathrm{~Hz}$; $-166.4,{ }^{1} J(\mathrm{P}, \mathrm{W})=251.6 \mathrm{~Hz} ;{ }^{4} J-$ $(\mathrm{P}, \mathrm{P})=4.1 \mathrm{~Hz})$. The assignment of the anti,anti-isomer $\mathbf{2 8}$ a was confirmed by a single-crystal Xray structure, depicted in Figure 4, that shows a tightened cyclopropanated phosphirane ring, ${ }^{[5,6]}(\mathrm{P}-\mathrm{C} 1.810$ (av); $\mathrm{C} 1-$ $\mathrm{C} 21.479(4))$ as compared to the terminal one $(\mathrm{P}-\mathrm{C} 1.829$ (av); $\mathrm{C} 1-\mathrm{C} 2 \quad 1.526(4))$ (Table 3).

This phosphirane, with spirofused cyclopropanes at both carbons, ${ }^{[5]}$ is similar to the PCC ring of 7 and 17a. Selected X-

that the second spirocyclopropane ring influences the phosphinidene addition, in that $\mathbf{2 2}$ gives a small amount of byproduct that structurally resembles $\mathbf{1 9}$. Before examining the influence of the catalyst, the special reactivity of the tetrasubstituted double bond in a bicyclopropylidene was compared with that of a vinyl substituent.

Reaction of $\mathbf{8}$ with an excess of ethenylbicyclopropylidene (25) in toluene at room temperature in the presence of $\mathrm{CuCl}$ gave after $18 \mathrm{~h}$ phospha[3]triangulane $\mathbf{2 6}$ (two isomers in a 1:1 ratio) and phosphirane 27 in a total yield of $89 \%$ (four isomers: 6:11:17:66) in a 1:9 ratio (Scheme 4). Both structure types were fully identified by NMR spectroscopy and show characteristic features, such as the about $40 \mathrm{ppm}$ more shielded ${ }^{31} \mathrm{P}$ NMR chemical shift for $\mathbf{2 7}$ that is

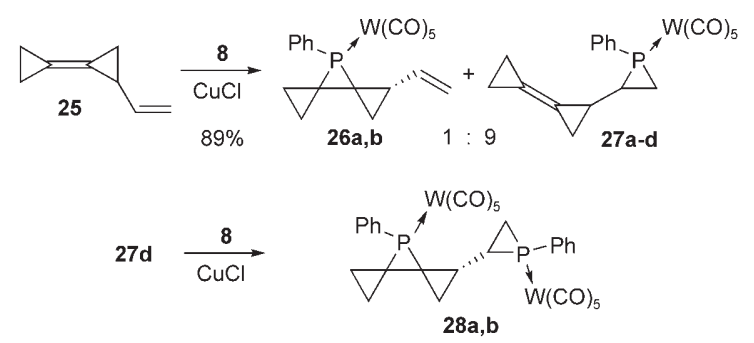

Scheme 4 . ray crystal structural data for these phospha[ $n]$ triangulanes and the previously reported parent $\mathbf{4} \mathbf{a}^{[4]}$ and $\mathbf{5 a} \mathbf{a}^{[5]}$ are summarized in Table 3. Phospha[7]triangulane $\mathbf{7}$ has a $\mathrm{P}-\mathrm{C}$ bond length of $1.820 \AA$ (av), which is comparable to the average length of the two unlike bonds (av 1.796 and $1.846 \AA$ ) of the phospha $[n]$ triangulanes with a terminal phosphirane ring (Table 1).

The additional spirofusion on the phosphirane ring is reflected in the NMR data (Tables 2 and 4) and most profoundly in the phosphorus chemical shift of $\delta^{31} \mathrm{P}-120$ to $-130 \mathrm{ppm}\left({ }^{1} \mathrm{~J}(\mathrm{P}, \mathrm{W})=250-260 \mathrm{~Hz}\right)$ that is deshielded by about 25 ppm compared with the terminal spirofused phosphirane complexes and by $55-65 \mathrm{ppm}$ from that of the parent 4a $(\delta-187.6 \mathrm{ppm}) .{ }^{[4]}$ The larger phospha $[n]$ triangulanes $(n=4)$ have the slightly more deshielded ${ }^{31} \mathrm{P}$ NMR resonances, thereby showing only a modest influence of the second sphere of spirocyclopropane rings. This influence is also reflected in the ${ }^{13} \mathrm{C}$ NMR chemical shifts of $\delta 23.5-26.5$ and $30.0-34.5 \mathrm{ppm}$ for the spirofused phosphirane carbons with first and second sphere spirocyclopropane rings, respectively. The effect is the strongest for phospha[7]triangulane complex $7^{[11]}$ with $\delta^{13} \mathrm{C} 34.5 \mathrm{ppm}$.

CuCl Catalysis: We return to the role of $\mathrm{CuCl}$ in the cycloaddition. Phosphiranes are the major products in all cases, 
Table 4. Selected NMR parameters for the phosphirane ring of phospha $[n]$ triangulanes.

\begin{tabular}{|c|c|c|c|c|c|c|c|c|c|c|c|c|}
\hline & $\mathbf{4 a}^{[5]}$ & $5 \mathbf{a}^{[5]}$ & $\boldsymbol{7}^{[11]}$ & $21 \mathrm{a}$ & $21 b$ & 23 & $26 a$ & $26 b$ & $28 a^{[a]}$ & $28 a^{[b]}$ & $28 b^{[a]}$ & $28 b^{[b]}$ \\
\hline${ }^{31} \mathrm{P}$ & -187.6 & -129.4 & -119.6 & -123.9 & -128.1 & -124.1 & -127.0 & -129.8 & -127.1 & -164.0 & -129.6 & -166.4 \\
\hline${ }^{1} J(\mathrm{P}, \mathrm{W})$ & 257.5 & 251.6 & 250.1 & 257.2 & 254.1 & 250.6 & 259.4 & 260.4 & 258.7 & 252.6 & 259.7 & 251.6 \\
\hline \multirow[t]{2}{*}{${ }^{13} \mathrm{C}(\mathrm{C} 1 / 2)$} & 10.7 & 26.0 & 34.5 & 30.1 & 30.0 & 32.3 & 31.0 & & 31.8 & 27.4 & 31.4 & 27.3 \\
\hline & & & & 24.8 & 25.3 & 23.5 & 26.5 & & 25.1 & 16.0 & 25.9 & 15.8 \\
\hline \multirow[t]{2}{*}{${ }^{1} J(\mathrm{C} 1 / 2, \mathrm{P})$} & 12.2 & 27.7 & 6.2 & 2.3 & 3.5 & 5.7 & 2.2 & & 3.8 & 16.2 & 5.3 & 15.9 \\
\hline & & & & - & - & - & - & & - & 11.6 & - & 11.4 \\
\hline
\end{tabular}

[a] Disubstituted phosphirane ring. [b] Terminal phosphirane ring.

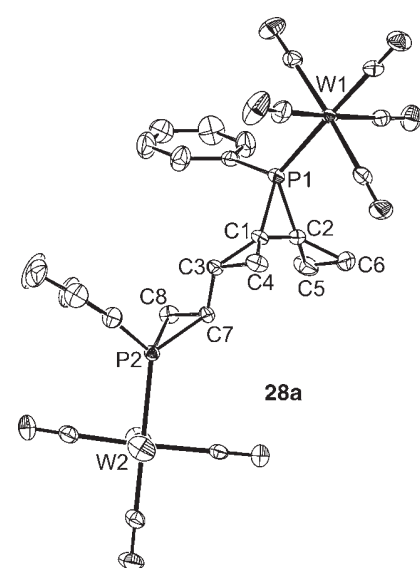

Figure 4. Displacement ellipsoid plot of 28 a with ellipsoids set at the $50 \%$ probability level. Hydrogen atoms are omitted for clarity. Selected bond lengths $[\AA]$ and angles [ ${ }^{\circ}$ ]: C3-C7 1.487(4); C3-C1-C4 60.97(18), C5-C2-C6 61.1(2).

suggesting that transient phosphinidene complex $\mathrm{Ph}-\mathrm{P}=$ $\mathrm{W}(\mathrm{CO})_{5}$ adds to the $\mathrm{C}=\mathrm{C}$ bond. However, there are exceptions, namely alkenes $\mathbf{2 2}$ and $\mathbf{1 8}$ with their full second sphere of spirocyclopropane rings at one and both ends of the double bond, respectively. They gave additional small amounts of the respective 2-phosphabicyclo[3.2.0]hept-1(5)enes 19 and 24. An X-ray crystal structure has been reported for 19. ${ }^{[11]}$ The NMR data of the two products are very similar. Characteristic are the ${ }^{31} \mathrm{P}$ and two ${ }^{13} \mathrm{C}$ chemical shifts for the ring C-P-C unit of 19 at $\delta^{31} \mathrm{P} 34.4\left({ }^{1} J(\mathrm{P}, \mathrm{W})\right.$ $238.4 \mathrm{~Hz}), \quad \delta^{13} \mathrm{C} \quad 34.9 \quad\left({ }^{1} J(\mathrm{P}, \mathrm{C}) \quad 39.7 \mathrm{~Hz}\right)$, and $142.5 \mathrm{ppm}$ $\left({ }^{1} J(\mathrm{P}, \mathrm{C}) 31.2 \mathrm{~Hz}\right)^{[11]}$ and those for 24 at $\delta^{31} \mathrm{P} 34.6\left({ }^{1} J(\mathrm{P}, \mathrm{W})\right.$ $234.7 \mathrm{~Hz}), \quad \delta^{13} \mathrm{C} \quad 33.1 \quad\left({ }^{1} J(\mathrm{P}, \mathrm{C}) \quad 40.2 \mathrm{~Hz}\right)$, and $140.3 \mathrm{ppm}$ $\left({ }^{1} J(\mathrm{P}, \mathrm{C}) 29.7 \mathrm{~Hz}\right)$. These alkenes, $\mathbf{1 8}$ and 22, but also $\mathbf{1 0}$ and 25, differ from the others in the fact that they react at room temperature within 18-20 h with the phosphinidene precursor 8 instead of the $55-60{ }^{\circ} \mathrm{C}^{[12]}$ which is usually needed for the $\mathrm{CuCl}$-catalyzed cycloadditions. Furthermore, the reactions executed at $55^{\circ} \mathrm{C}$ occur rather fast $(1-2 \mathrm{~h})$ while the slower second cycloaddition for $\mathbf{1 6 b}(3 \mathrm{~h})$ and $\mathbf{2 7 d}(6.5 \mathrm{~h})$ differ from each other. These observations suggest that the elimination of the phosphinidene complex from the precursor is not necessarily the rate-limiting step, but that the alkene may be involved as well. ${ }^{[19]}$ This notion concurs with a computational analysis, ${ }^{[20]}$ which suggested that the alkene or aromatic solvent interacts with $\mathrm{CuCl}$ and that this complex dissociates the precursor to formally give
$\left[\mathrm{PhP}(\mathrm{Cl}) \mathrm{W}(\mathrm{CO})_{5}\right]-\mathrm{Cu}-\mathrm{L}(\mathrm{L}=$ alkene or solvent $)$ as the active reagent. Phosphirane formation then occurs in an $\mathrm{S}_{\mathrm{N}} 2$-type addition with a concurrent "chloride shuttle" back to the $\mathrm{Cu}$ ion to regenerate the $\mathrm{CuCl}-\mathrm{L}$ complex. While the products for the uncatalyzed and $\mathrm{CuCl}$-catalyzed reactions are usually the same, they can differ ${ }^{[22]}$ and this was also the case with the alkenes $\mathbf{1 8}$ and 22. Indeed, phosphatriangulane $\mathbf{7}$ is the sole product formed from 18 in the absence of $\mathrm{CuCl}^{[11]}$ There are two aspects, $\left[\mathrm{PhP}(\mathrm{Cl}) \mathrm{W}(\mathrm{CO})_{5}\right]-\mathrm{Cu}-\mathrm{L}$ is less reactive and bulkier than $\mathrm{R}-\mathrm{P}=\mathrm{W}(\mathrm{CO})_{5}$ and thus more sensitive to steric constraints, which is the case here. We presume that the $\mathrm{C}=\mathrm{C}$ bond interacts with the $\mathrm{CuCl}-\mathrm{L}$ ligated phosphinidene complex under expulsion of the $\mathrm{CuCl}-\mathrm{L}$ complex, forming zwitterion 29 (Scheme 5). Analogous $\sigma$-complexes

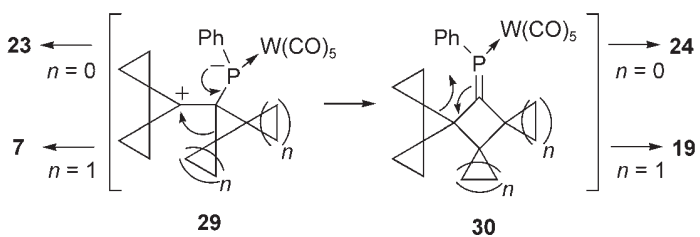

Scheme 5 .

were identified computationally to precede the formation of the 1,4-addition and $\mathrm{C}-\mathrm{H}$ insertion products of azulenes. ${ }^{[23]}$ Branched phospha $[n]$ triangulanes $\mathbf{7}$ and $\mathbf{2 3}$ will result upon ring closure of the zwitterion, but steric factors may facilitate the well-known cyclopropylcarbinyl to cyclobutyl ring enlargement ${ }^{[24]}$ to form phosphaalkene 30. The ring expansion preferentially involves the more highly substituted cyclopropane ring, because in this manner the cationic charge can be delocalized the best. ${ }^{[25,26]}$ The reactive $\mathrm{P}=\mathrm{C}$ bond of 30 enables a subsequent [1,3]-sigmatropic shift ${ }^{[27]}$ that gives the 2-phosphabicyclo[3.2.0]heptenes $\mathbf{1 9}$ and $\mathbf{2 4}$.

\section{Conclusion}

Novel, linear and branched mono- and diphospha[ $n]$ triangulanes have been synthesized in high yields by the $\mathrm{CuCl}$-catalyzed phosphinidene addition to spirocyclopropanated methylenecyclopropanes and bicyclopropylidenes. Single crystal X-ray structure analyses of these remarkably stable products revealed a tightening of the phosphirane ring on spirocyclopropanation. The effect of spirofusion on the electronic 
properties of these strained phosphacycles is apparent from the NMR features that show deshielded chemical shifts for the phosphorus and carbon ring atoms. Steric factors play a role when the double bond carries a second sphere of spirocyclopropane rings and causes the formation of 2phosphabicyclo[3.2.0]heptenes as byproducts. The latter are explained to result from addition of the $\left[\mathrm{PhP}(\mathrm{Cl}) \mathrm{W}(\mathrm{CO})_{5}\right]-$ $\mathrm{Cu}-\mathrm{L}(\mathrm{L}=$ alkene or solvent) reagent to the spirocyclopropanated bicyclopropylidene to give an intermediate zwitterion, which can undergo a cyclopropylcarbinyl to cyclobutyl ring expansion followed by a [1,3]-sigmatropic shift.

\section{Experimental Section}

All experiments were performed under an atmosphere of dry nitrogen. Solids were dried in vacuo and liquids were distilled (under $\mathrm{N}_{2}$ ) prior to use. Solvents were used as purchased, except for toluene, which was distilled over sodium. NMR spectra were recorded (at $298 \mathrm{~K}$ ) on a Bruker Advance $250\left({ }^{31} \mathrm{P} ; 85 \% \mathrm{H}_{3} \mathrm{PO}_{4}\right)$ and a MSL $400\left({ }^{1} \mathrm{H},{ }^{13} \mathrm{C}\right)$ and referenced internally to residual solvent resonances $\left({ }^{1} \mathrm{H}: \delta 7.25 \mathrm{ppm}\left(\mathrm{CHCl}_{3}\right)\right.$, ${ }^{13} \mathrm{C}\left\{{ }^{1} \mathrm{H}\right\}$ : $77.0 \mathrm{ppm}\left(\mathrm{CDCl}_{3}\right)$. Isomeric ratios were determined by ${ }^{31} \mathrm{P} \mathrm{NMR}$ spectroscopy. IR spectra were recorded on a Mattson-6030 Galaxy FT-IR spectrophotometer, and high-resolution mass spectra (HR-MS) were measured on a Finnigan Mat 900 mass spectrometer operating at an ionization potential of $70 \mathrm{eV}$. Melting points were measured on samples in unsealed capillaries and are uncorrected. $\mathrm{CuCl}$ ( $99 \%$ purity) was purchased from Acros and stored under nitrogen before use. (5,6-Dimethyl2,3-bis(methoxycarbonyl)-7-phenyl-7-phosphanorbornadiene) pentacarbonyltungsten $(\mathbf{8}),{ }^{[9,13]}$ methylenespiropentane $(\mathbf{9}),{ }^{[28]}$ 1-methylenedispiro[2.0.2.1] heptane (10),${ }^{[29]}$ 7-methylenedispiro[2.0.2.1] heptane $(\mathbf{1 1}),{ }^{[29,30]} 1,4$ bismethylenespiropentane $(\mathbf{1 5}),{ }^{[31]}$ perspirocyclopropanated bicyclopropylidene $\mathbf{1 8}{ }^{[3]}$ cyclopropylidenespiropentane $(\mathbf{2 0}),{ }^{[32]}$ 7-cyclopropylidenedispiro[2.0.2.1]heptane $(\mathbf{2 2}),{ }^{[30]}$ and ethenylbicyclopropylidene $(\mathbf{2 5})^{[33]}$ were prepared according to literature procedures.

(1-Phenyl-1-phosphadispiro[2.0.2.1]hept-1-yl)pentacarbonyltungsten (12): Complex 8 (360 mg, $0.55 \mathrm{mmol}$ ), methylenespiropentane (9) (132 mg, $1.65 \mathrm{mmol})$ and $\mathrm{CuCl}(10 \mathrm{mg}, 0.1 \mathrm{mmol})$ were heated in toluene $(4 \mathrm{~mL})$ at $55^{\circ} \mathrm{C}$ for $1 \mathrm{~h}$. Evaporation to dryness and chromatography of the residue over silica gel eluting with pentane/dichloromethane 19:1 gave 12a and $\mathbf{b}$ in a $5: 4$ ratio $(220 \mathrm{mg}, 78 \%)$ as a pale yellow oil. Fractional recrystallization from pentane at $-80^{\circ} \mathrm{C}$ followed by recrystallization at $-20^{\circ} \mathrm{C}$ afforded colorless crystals of the anti-isomer $\mathbf{1 2} \mathbf{a}$.

anti-(1R,3S)- and (1S,3R)-Isomer 12a: m.p. $\quad 64^{\circ} \mathrm{C} ; \quad{ }^{1} \mathrm{H} N M R$ $\left(400.13 \mathrm{MHz}, \mathrm{CDCl}_{3}\right): \delta=0.49-0.55(\mathrm{~m}, 1 \mathrm{H} ; \mathrm{CH}), 0.65-0.71(\mathrm{~m}, 1 \mathrm{H}$; $\mathrm{CH}), 0.85-0.91(\mathrm{~m}, 1 \mathrm{H} ; \mathrm{CH}), 0.92-0.96(\mathrm{~m}, 1 \mathrm{H} ; \mathrm{CH}), 1.39\left(\mathrm{dd},{ }^{2} \mathrm{~J}(\mathrm{H}, \mathrm{H})=\right.$ $\left.4.1,{ }^{3} J(\mathrm{H}, \mathrm{P})=6.2 \mathrm{~Hz}, 1 \mathrm{H}, \mathrm{PCCH}\right), 1.77\left(\mathrm{dd},{ }^{2} J(\mathrm{H}, \mathrm{H})=4.1,{ }^{3} J(\mathrm{H}, \mathrm{P})=\right.$ $10.2 \mathrm{~Hz}, 1 \mathrm{H}, \mathrm{PCCH}), 1.79\left(\mathrm{~d},{ }^{2} J(\mathrm{H}, \mathrm{H})=7.6 \mathrm{~Hz}, 1 \mathrm{H} ; \mathrm{PCH}\right), 1.99(\mathrm{dd}$, $\left.{ }^{2} J(\mathrm{H}, \mathrm{H})=7.6,{ }^{2} J(\mathrm{H}, \mathrm{P})=7.1 \mathrm{~Hz}, 1 \mathrm{H} ; \mathrm{PCH}\right), 7.30-7.38 \mathrm{ppm}(\mathrm{m}, 5 \mathrm{H} ; \mathrm{PhH})$; $\left.{ }^{13} \mathrm{C}{ }^{1} \mathrm{H}\right\}$ NMR $\left(100.62 \mathrm{MHz}, \mathrm{CDCl}_{3}\right): \delta=5.2\left(\mathrm{~d},{ }^{3} J(\mathrm{C}, \mathrm{P})=4.5 \mathrm{~Hz} ; \mathrm{CH}_{2}\right)$, $5.9\left(\mathrm{~s} ; \mathrm{CH}_{2}\right), 17.0\left(\mathrm{~d},{ }^{1} \mathrm{~J}(\mathrm{C}, \mathrm{P})=\sim 6 \mathrm{~Hz} ; \mathrm{PCH}_{2}\right), 17.1\left(\mathrm{~s} ; \mathrm{PCCH}_{2}\right), 18.5(\mathrm{~d}$, $\left.{ }^{2} J(\mathrm{C}, \mathrm{P})=5.3 \mathrm{~Hz} ; \mathrm{PCC}\right), 26.5\left(\mathrm{~d},{ }^{1} J(\mathrm{C}, \mathrm{P})=6.1 \mathrm{~Hz} ; \mathrm{PC}\right), 128.6\left(\mathrm{~d},{ }^{3} J(\mathrm{C}, \mathrm{P})=\right.$ $10.5 \mathrm{~Hz} ; m-\mathrm{Ph}), 130.2\left(\mathrm{~d},{ }^{4} J(\mathrm{C}, \mathrm{P})=2.5 \mathrm{~Hz} ; p-\mathrm{Ph}\right), 131.4\left(\mathrm{~d},{ }^{2} J(\mathrm{C}, \mathrm{P})=\right.$ $13.4 \mathrm{~Hz} ; o-\mathrm{Ph}), 132.5\left(\mathrm{~d},{ }^{1} J(\mathrm{C}, \mathrm{P})=25.6 \mathrm{~Hz}\right.$; ipso-Ph), $195.6\left(\mathrm{~d},{ }^{2} J(\mathrm{C}, \mathrm{P})=\right.$ $8.4,{ }^{1} J(\mathrm{C}, \mathrm{W})=125.5 \mathrm{~Hz}$; cis-CO), $197.9 \mathrm{ppm}\left(\mathrm{d},{ }^{2} J(\mathrm{C}, \mathrm{P})=30.0 \mathrm{~Hz}\right.$; trans$\mathrm{CO}) ;{ }^{31} \mathrm{P} \mathrm{NMR} \quad\left(101.25 \mathrm{MHz}, \quad \mathrm{CDCl}_{3}\right): \delta=-146.4 \mathrm{ppm} \quad\left({ }^{1} J(\mathrm{P}, \mathrm{W})=\right.$ $257.2 \mathrm{~Hz}$ ); IR (KBr): $\tilde{v}=1932\left(\mathrm{~s} / \mathrm{br}, \mathrm{CO}_{\mathrm{eq}}\right), 1983\left(\mathrm{w}, \mathrm{CO}_{\mathrm{eq}}\right), 2074 \mathrm{~cm}^{-1}(\mathrm{w}$, $\left.\mathrm{CO}_{\mathrm{ax}}\right) ; \mathrm{MS}(70 \mathrm{eV}): \mathrm{m} / z(\%): 512(8)[M]^{+}, 484(4)[M-\mathrm{CO}]^{+}, 456(9)$ $[M-2 \mathrm{CO}]^{+}, 428(8)[M-3 \mathrm{CO}]^{+}, 400(32)[M-4 \mathrm{CO}]^{+}, 372 \quad(100)$ $[M-5 \mathrm{CO}]^{+}$; HR-MS (EI): $m / z$ : calcd for $\mathrm{C}_{17} \mathrm{H}_{13} \mathrm{O}_{5} \mathrm{P}^{184} \mathrm{~W}: 512.00104$; found: 511.99819 .

syn-(1R,3R)- and (1S,3S)-Isomer 12 b: ${ }^{1} \mathrm{H}$ NMR (400.13 $\left.\mathrm{MHz}, \mathrm{CDCl}_{3}\right)$ : $\delta=0.85-0.91(\mathrm{~m}, 1 \mathrm{H} ; \mathrm{CH}), 0.91-0.97(\mathrm{~m}, 1 \mathrm{H} ; \mathrm{CH}), 1.08-1.14(\mathrm{~m}, 1 \mathrm{H}$; $\mathrm{CH}), 1.20-1.25$ (m, 1 H; CH), 1.58-1.62 (m, 2H; PCH, PCCH), 1.68 (dd, $\left.{ }^{2} J(\mathrm{H}, \mathrm{H})=4.0,{ }^{3} J(\mathrm{H}, \mathrm{P})=11.1 \mathrm{~Hz}, 1 \mathrm{H} ; \mathrm{PCCH}\right), 2.02\left(\mathrm{dd},{ }^{2} J(\mathrm{H}, \mathrm{H})=7.4,{ }^{2} J-\right.$
$(\mathrm{H}, \mathrm{P})=7.1 \mathrm{~Hz}, 1 \mathrm{H} ; \mathrm{PCH}), 7.39-7.44(\mathrm{~m}, 3 \mathrm{H} ; m-\mathrm{Ph} H, p-\mathrm{Ph} H), 7.52-$ $7.57 \mathrm{ppm}(\mathrm{m}, 2 \mathrm{H} ; o-\mathrm{Ph} H) ;{ }^{13} \mathrm{C}\left\{{ }^{1} \mathrm{H}\right\}$ NMR $\left(100.62 \mathrm{MHz}, \mathrm{CDCl}_{3}\right): \delta=5.2$ $\left(\mathrm{d},{ }^{3} \mathrm{~J}(\mathrm{C}, \mathrm{P})=1.8 \mathrm{~Hz} ; \mathrm{CH}_{2}\right), 6.2\left(\mathrm{~d},{ }^{3} \mathrm{~J}(\mathrm{C}, \mathrm{P})=3.4 \mathrm{~Hz} ; \mathrm{CH}_{2}\right), 16.4\left(\mathrm{~d},{ }^{2} J-\right.$ $\left.(\mathrm{C}, \mathrm{P})=3.5 \mathrm{~Hz} ; \mathrm{PCCH}_{2}\right), 17.4\left(\mathrm{~d},{ }^{1} J(\mathrm{C}, \mathrm{P})=6.1 \mathrm{~Hz} ; \mathrm{PCH}_{2}\right), 19.3(\mathrm{~s} ; \mathrm{PCC})$, $25.7\left(\mathrm{~d},{ }^{1} J(\mathrm{C}, \mathrm{P})=7.1 \mathrm{~Hz} ; \mathrm{PC}\right), 128.7\left(\mathrm{~d},{ }^{3} J(\mathrm{C}, \mathrm{P})=10.4 \mathrm{~Hz} ; m-\mathrm{Ph}\right), 130.2$ $\left(\mathrm{d},{ }^{4} J(\mathrm{C}, \mathrm{P})=2.3 \mathrm{~Hz} ; p-\mathrm{Ph}\right), 131.4\left(\mathrm{~d},{ }^{2} J(\mathrm{C}, \mathrm{P})=13.1 \mathrm{~Hz} ; o-\mathrm{Ph}\right), 134.6\left(\mathrm{~d},{ }^{1} J-\right.$ $(\mathrm{C}, \mathrm{P})=25.4 \mathrm{~Hz}$; ipso-Ph), $195.7\left(\mathrm{~d},{ }^{2} J(\mathrm{C}, \mathrm{P})=8.3,{ }^{1} \mathrm{~J}(\mathrm{C}, \mathrm{W})=125.6 \mathrm{~Hz}\right.$; cisCO), $197.8 \mathrm{ppm}\left(\mathrm{d},{ }^{2} J(\mathrm{C}, \mathrm{P})=29.8 \mathrm{~Hz}\right.$; trans-CO); ${ }^{31} \mathrm{P}$ NMR $(101.25 \mathrm{MHz}$, $\left.\mathrm{CDCl}_{3}\right): \delta=-152.3 \mathrm{ppm}\left({ }^{1} \mathrm{~J}(\mathrm{P}, \mathrm{W})=254.1 \mathrm{~Hz}\right)$.

(1-Phenyl-1-phosphatrispiro[2.0.0.2.1.1]non-1-yl)pentacarbonyltungsten (13): Complex 8 (450 mg, $0.69 \mathrm{mmol})$, 1-methylenedispiro[2.0.2.1] heptane (10) $(183 \mathrm{mg}, 1.72 \mathrm{mmol})$, and $\mathrm{CuCl}(10 \mathrm{mg}, 0.1 \mathrm{mmol})$ were stirred in toluene $(4 \mathrm{~mL})$ at room temperature for $18 \mathrm{~h}$. Evaporation to dryness and chromatography of the residue over silica gel eluting with pentane/ dichloromethane 9:1 gave a 4:50:10:36 mixture of isomers 13a, b, c and d $(350 \mathrm{mg}, 94 \%)$ as a pale yellow oil. Recrystallization from pentane at $-80^{\circ} \mathrm{C}$ afforded a 10:7 isomeric mixture of the main isomers $\mathbf{1 3 b}$ and $13 \mathrm{~d}$.

Compound 13a: ${ }^{31} \mathrm{P}$ NMR (101.25 MHz, $\left.\mathrm{CDCl}_{3}\right): \delta=-147.3 \mathrm{ppm}\left({ }^{1} J-\right.$ $(\mathrm{P}, \mathrm{W})=258.2 \mathrm{~Hz})$.

anti-(1R,3S,4R)- and $(1 S, 3 R, 4 S)$-Isomer $13 \mathrm{~b}:{ }^{1} \mathrm{H}$ NMR $(400.13 \mathrm{MHz}$, $\left.\mathrm{CDCl}_{3}\right): \delta=0.76-0.96\left(\mathrm{~m}, 5 \mathrm{H} ; \mathrm{CH}_{2}, \mathrm{PCCCH}\right), 1.23-1.27(\mathrm{~m}, 2 \mathrm{H} ; \mathrm{PCCH}$, $\mathrm{PCCCH}), 1.68-1.73(\mathrm{~m}, 1 \mathrm{H} ; \mathrm{PCH}), 1.81\left(\mathrm{dd},{ }^{2} J(\mathrm{H}, \mathrm{H})=4.4,{ }^{3} J(\mathrm{H}, \mathrm{P})=\right.$ $10.0 \mathrm{~Hz}, 1 \mathrm{H} ; \mathrm{PCCH}), 2.02\left(\mathrm{dd},{ }^{2} J(\mathrm{H}, \mathrm{H})=7.4,{ }^{2} J(\mathrm{H}, \mathrm{P})=7.1 \mathrm{~Hz}, 1 \mathrm{H}\right.$; $\left.\mathrm{PCH}), \quad 7.34-7.45 \mathrm{ppm}(\mathrm{m}, 5 \mathrm{H} ; \mathrm{PhH}) ;{ }^{13} \mathrm{C}^{1} \mathrm{H}\right\} \quad \mathrm{NMR}(100.62 \mathrm{MHz}$, $\left.\mathrm{CDCl}_{3}\right): \delta=4.0\left(\mathrm{~s} ; \mathrm{CH}_{2}\right), 5.1\left(\mathrm{~d},{ }^{4} J(\mathrm{C}, \mathrm{P})=1.2 \mathrm{~Hz} ; \mathrm{CH}_{2}\right), 11.7\left(\mathrm{~d},{ }^{3} J(\mathrm{C}, \mathrm{P})=\right.$ $4.6 \mathrm{~Hz} ; \mathrm{PCCCH}), 14.7(\mathrm{~s} ; \mathrm{PCCC}), 16.1(\mathrm{~s} ; \mathrm{PCCH}), 16.7\left(\mathrm{~d},{ }^{1} J(\mathrm{C}, \mathrm{P})=\right.$ $\left.6.3 \mathrm{~Hz} ; \mathrm{PCH}_{2}\right), 22.4\left(\mathrm{~d},{ }^{2} J(\mathrm{C}, \mathrm{P})=8.8 \mathrm{~Hz} ; \mathrm{PCC}\right), 26.7\left(\mathrm{~d},{ }^{1} J(\mathrm{C}, \mathrm{P})=6.7 \mathrm{~Hz}\right.$; $\mathrm{PC}), 128.5\left(\mathrm{~d},{ }^{3} J(\mathrm{C}, \mathrm{P})=10.8 \mathrm{~Hz} ; m-\mathrm{Ph}\right), 130.3\left(\mathrm{~d},{ }^{4} J(\mathrm{C}, \mathrm{P})=2.7 \mathrm{~Hz} ; p-\mathrm{Ph}\right)$, $131.7\left(\mathrm{~d},{ }^{2} J(\mathrm{C}, \mathrm{P})=13.9 \mathrm{~Hz} ; o-\mathrm{Ph}\right), 132.0\left(\mathrm{~d},{ }^{1} J(\mathrm{C}, \mathrm{P})=24.9 \mathrm{~Hz} ;\right.$ ipso-Ph $)$, $195.7\left(\mathrm{~d},{ }^{2} J(\mathrm{C}, \mathrm{P})=8.4,{ }^{1} J(\mathrm{C}, \mathrm{W})=125.5 \mathrm{~Hz} ;\right.$ cis-CO $), 197.7 \mathrm{ppm}\left(\mathrm{d},{ }^{2} J-\right.$ $(\mathrm{C}, \mathrm{P})=30.2 \mathrm{~Hz} ; \quad$ trans-CO $) ; \quad{ }^{31} \mathrm{P} \mathrm{NMR} \quad\left(101.25 \mathrm{MHz}, \quad \mathrm{CDCl}_{3}\right): \quad \delta=$ $-152.2 \mathrm{ppm}\left({ }^{1} J(\mathrm{P}, \mathrm{W})=258.1 \mathrm{~Hz}\right)$; MS $(70 \mathrm{eV})$ : (mixture of $\mathbf{1 3 b}$ and $\left.\mathbf{1 3 d}\right)$ : $m / z(\%): 538(6)[M]^{+}, 482(2)[M-2 \mathrm{CO}]^{+}, 454(5)[M-3 \mathrm{CO}]^{+}, 426(22)$ $[M-4 \mathrm{CO}]^{+}, 398(88)[M-5 \mathrm{CO}]^{+}$; HR-MS (EI) calcd for $\mathrm{C}_{19} \mathrm{H}_{15} \mathrm{O}_{5} \mathrm{P}^{184} \mathrm{~W}$ : 538.01672; found: 538.01741 .

Compound 13c: ${ }^{31} \mathrm{P}$ NMR $\left(101.25 \mathrm{MHz}, \mathrm{CDCl}_{3}\right): \delta=-152.3 \mathrm{ppm}\left({ }^{1} J-\right.$ $(\mathrm{P}, \mathrm{W})=254.4 \mathrm{~Hz})$.

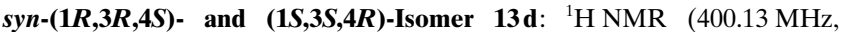
$\left.\mathrm{CDCl}_{3}\right): \delta=0.69-0.73(\mathrm{~m}, 1 \mathrm{H}, \mathrm{CH}), 0.76-0.96\left(\mathrm{~m}, 3 \mathrm{H} ; \mathrm{CH}_{2}\right), 1.44\left(\mathrm{dd},{ }^{2} J-\right.$ $\left.(\mathrm{H}, \mathrm{H})=4.3,{ }^{3} J(\mathrm{H}, \mathrm{P})=1.7 \mathrm{~Hz}, 1 \mathrm{H} ; \mathrm{PCCH}\right), 1.46,1.47,1.60,1.61$ (AB type, $\left.{ }^{2} J(\mathrm{H}, \mathrm{H})=4.3 \mathrm{~Hz}, 2 \mathrm{H} ; \mathrm{PCCCH}_{2}\right), 1.68-1.73(\mathrm{~m}, 2 \mathrm{H} ; \mathrm{PCH}, \mathrm{PCCH})$, $1.88\left(\mathrm{dd},{ }^{2} J(\mathrm{H}, \mathrm{H})=7.3,{ }^{2} J(\mathrm{H}, \mathrm{P})=7.1 \mathrm{~Hz}, 1 \mathrm{H} ; \mathrm{PCH}\right), 7.34-7.45(\mathrm{~m}, 3 \mathrm{H}$; $m-\mathrm{Ph} H, \quad p-\mathrm{Ph} H), \quad 7.52-7.57 \mathrm{ppm} \quad(\mathrm{m}, \quad 2 \mathrm{H} ; \quad o-\mathrm{Ph} H) ;{ }^{13} \mathrm{C}\left\{{ }^{1} \mathrm{H}\right\} \quad$ NMR $\left(100.62 \mathrm{MHz}, \mathrm{CDCl}_{3}\right): \delta=4.2\left(\mathrm{~s} ; \mathrm{CH}_{2}\right), 4.8\left(\mathrm{~d},{ }^{4} \mathrm{~J}(\mathrm{C}, \mathrm{P})=2.1 \mathrm{~Hz} ; \mathrm{CH}_{2}\right)$, $12.7\left(\mathrm{~d},{ }^{3} J(\mathrm{C}, \mathrm{P})=3.9 \mathrm{~Hz} ; \mathrm{PCCCH}\right), 14.5\left(\mathrm{~d},{ }^{3} \mathrm{~J}(\mathrm{C}, \mathrm{P})=3.2 \mathrm{~Hz} ; \mathrm{PCCC}\right), 15.2$ $\left(\mathrm{d},{ }^{2} J(\mathrm{C}, \mathrm{P})=3.6 \mathrm{~Hz} ; \mathrm{PCCH}\right), 16.4\left(\mathrm{~d},{ }^{1} J(\mathrm{C}, \mathrm{P})=6.4 \mathrm{~Hz} ; \mathrm{PCH}_{2}\right), 23.1$ (s; $\mathrm{PCC}), 25.6\left(\mathrm{~d},{ }^{1} J(\mathrm{C}, \mathrm{P})=8.6 \mathrm{~Hz} ; \mathrm{PC}\right), 128.7\left(\mathrm{~d},{ }^{3} J(\mathrm{C}, \mathrm{P})=10.4 \mathrm{~Hz} ; m-\mathrm{Ph}\right)$, $130.2\left(\mathrm{~d},{ }^{4} J(\mathrm{C}, \mathrm{P})=2.4 \mathrm{~Hz} ; p-\mathrm{Ph}\right), 131.5\left(\mathrm{~d},{ }^{2} J(\mathrm{C}, \mathrm{P})=13.1 \mathrm{~Hz} ; o-\mathrm{Ph}\right), 134.6$ $\left(\mathrm{d}, \quad{ }^{1} J(\mathrm{C}, \mathrm{P})=25.3 \mathrm{~Hz} ; \quad\right.$ ipso- $\left.\mathrm{Ph}\right), \quad 195.7 \quad\left(\mathrm{~d}, \quad{ }^{2} J(\mathrm{C}, \mathrm{P})=8.3, \quad{ }^{1} J(\mathrm{C}, \mathrm{W})=\right.$ $125.5 \mathrm{~Hz}$; cis-CO), $197.9 \mathrm{ppm}\left(\mathrm{d},{ }^{2} J(\mathrm{C}, \mathrm{P})=30.0 \mathrm{~Hz}\right.$; trans-CO $) ;{ }^{31} \mathrm{P}$ NMR $\left(101.25 \mathrm{MHz}, \mathrm{CDCl}_{3}\right): \delta=-154.9 \mathrm{ppm}\left({ }^{1} \mathrm{~J}(\mathrm{P}, \mathrm{W})=255.5 \mathrm{~Hz}\right)$.

(1-Phenyl-1-phosphatrispiro[2.0.2.0.2.0]non-1-yl)pentacarbonyltungsten (14): Complex 8 (400 mg, $0.61 \mathrm{mmol})$, 7-methylenedispiro[2.0.2.1]heptane (11) $(97 \mathrm{mg}, 0.92 \mathrm{mmol})$ and $\mathrm{CuCl}(10 \mathrm{mg}, 0.1 \mathrm{mmol})$ were heated in toluene $(4 \mathrm{~mL})$ at $55^{\circ} \mathrm{C}$ for $1 \mathrm{~h}$. Evaporation to dryness and chromatography of the residue over silica gel eluting with pentane/dichloromethane 9:1 gave $14(290 \mathrm{mg}, 88 \%)$ as a pale yellow oil. Recrystallization from pentane at $-20^{\circ} \mathrm{C}$ afforded colorless crystals. M.p. $61-62{ }^{\circ} \mathrm{C} ;{ }^{1} \mathrm{H}$ NMR $\left(400.13 \mathrm{MHz}, \mathrm{CDCl}_{3}\right): \delta=0.34-0.38(\mathrm{~m}, 1 \mathrm{H} ; \mathrm{CH}), 0.57-0.62(\mathrm{~m}, 1 \mathrm{H}$; $\mathrm{CH}), 0.62-0.66(\mathrm{~m}, 1 \mathrm{H} ; \mathrm{CH}), 0.70-0.73(\mathrm{~m}, 1 \mathrm{H} ; \mathrm{CH}), 0.73-0.76(\mathrm{~m}, 1 \mathrm{H}$; $\mathrm{CH}), 0.88-0.95(\mathrm{~m}, 2 \mathrm{H} ; \mathrm{CH}), 1.20-1.30(\mathrm{~m}, 1 \mathrm{H} ; \mathrm{CH}), 1.62\left(\mathrm{dd},{ }^{2} J(\mathrm{H}, \mathrm{H})=\right.$ $\left.7.8,{ }^{2} J(\mathrm{H}, \mathrm{P})=0.9 \mathrm{~Hz}, 1 \mathrm{H} ; \mathrm{PCH}\right), 2.09\left(\mathrm{dd},{ }^{2} J(\mathrm{H}, \mathrm{H})=7.8,{ }^{2} J(\mathrm{H}, \mathrm{P})=7.0 \mathrm{~Hz}\right.$, $1 \mathrm{H} ; \mathrm{PCH}), 7.36-7.48 \mathrm{ppm}(\mathrm{m}, 5 \mathrm{H} ; \mathrm{PhH}) ;{ }^{13} \mathrm{C}\left\{{ }^{1} \mathrm{H}\right\}$ NMR $(100.62 \mathrm{MHz}$, $\left.\mathrm{CDCl}_{3}\right): \delta=4.4\left(\mathrm{~d},{ }^{3} J(\mathrm{C}, \mathrm{P})=3.8 \mathrm{~Hz} ; \mathrm{CH}_{2}\right), 4.7,5.0\left(2 \mathrm{~s} ; \mathrm{CH}_{2}\right), 5.9\left(\mathrm{~d},{ }^{3} J-\right.$ $\left.(\mathrm{C}, \mathrm{P})=2.8 \mathrm{~Hz} ; \mathrm{CH}_{2}\right), 15.5\left(\mathrm{~d},{ }^{1} J(\mathrm{C}, \mathrm{P})=5.7 \mathrm{~Hz} ; \mathrm{PCH}_{2}\right), 22.5(\mathrm{~s} ; \mathrm{PCC})$, $22.5\left(\mathrm{~d},{ }^{2} J(\mathrm{C}, \mathrm{P})=5.7 \mathrm{~Hz} ; \mathrm{PCC}\right), 29.6\left(\mathrm{~d},{ }^{1} J(\mathrm{C}, \mathrm{P})=10.1 \mathrm{~Hz} ; \mathrm{PC}\right), 128.6(\mathrm{~d}$, $\left.{ }^{3} J(\mathrm{C}, \mathrm{P})=10.4 \mathrm{~Hz} ; m-\mathrm{Ph}\right), 130.2\left(\mathrm{~d},{ }^{4} J(\mathrm{C}, \mathrm{P})=2.3 \mathrm{~Hz} ; p-\mathrm{Ph}\right), 131.3\left(\mathrm{~d},{ }^{2} J-\right.$ 
$(\mathrm{C}, \mathrm{P})=13.1 \mathrm{~Hz} ; o-\mathrm{Ph}), 132.9\left(\mathrm{~d},{ }^{1} J(\mathrm{C}, \mathrm{P})=25.9 \mathrm{~Hz} ;\right.$ ipso-Ph $), 195.7\left(\mathrm{~d},{ }^{2} J-\right.$ $(\mathrm{C}, \mathrm{P})=8.3,{ }^{1} J(\mathrm{C}, \mathrm{W})=125.5 \mathrm{~Hz} ;$ cis-CO $), 198.0 \mathrm{ppm}\left(\mathrm{d},{ }^{2} J(\mathrm{C}, \mathrm{P})=29.8 \mathrm{~Hz}\right.$; trans-CO); ${ }^{31} \mathrm{P}$ NMR $\left(101.25 \mathrm{MHz}, \mathrm{CDCl}_{3}\right): \delta=-145.2 \mathrm{ppm}\left({ }^{1} J(\mathrm{P}, \mathrm{W})=\right.$ $252.1 \mathrm{~Hz}$ ); IR (KBr): $\tilde{v}=1917$ (s/br, $\left.\mathrm{CO}_{\mathrm{eq}}\right), 1983\left(\mathrm{w}, \mathrm{CO}_{\mathrm{eq}}\right), 2070 \mathrm{~cm}^{-1}(\mathrm{~m}$, $\left.\mathrm{CO}_{\mathrm{ax}}\right) ; \mathrm{MS}(70 \mathrm{eV}): \mathrm{m} / z(\%): 538(10)\left[\mathrm{M}^{+}, 482(3)[M-2 \mathrm{CO}]^{+}, 454\right.$ (16) $[M-3 \mathrm{CO}]^{+}, 426(28)[M-4 \mathrm{CO}]^{+}$; HR-MS (EI): $\mathrm{m} / z$ : calcd for $\mathrm{C}_{19} \mathrm{H}_{15} \mathrm{O}_{5} \mathrm{P}^{184} \mathrm{~W}$ : 538.01672; found: 538.01581; elemental analysis calcd (\%) for $\mathrm{C}_{19} \mathrm{H}_{15} \mathrm{O}_{5} \mathrm{P}^{184} \mathrm{~W}: \mathrm{C} 42.41, \mathrm{H} 2.81$; found: $\mathrm{C} 41.68, \mathrm{H} 2.89$.

(1-Phenyl-1-phospha-5-methylenedispiro[2.0.2.1] hept-1-yl)pentacarbonyltungsten (16): Complex 8 ( $450 \mathrm{mg}, 0.69 \mathrm{mmol})$, rac-1,4-dimethylenespiropentane (15) $(191 \mathrm{mg}, 2.07 \mathrm{mmol})$ and $\mathrm{CuCl}(10 \mathrm{mg}, 0.1 \mathrm{mmol})$ were heated in toluene $(4 \mathrm{~mL})$ at $55^{\circ} \mathrm{C}$ for $2 \mathrm{~h}$. Evaporation to dryness and chromatography of the residue over silica gel eluting with pentane/dichloromethane 19:1 gave a 12:39:31:18 mixture of isomers of 16a, b, c and $\mathbf{d}(300 \mathrm{mg}, 83 \%)$ as a pale yellow oil. Compound $\mathbf{1 6 b}$ could be separated by sequential column chromatography over silica gel eluting with pentane/dichloromethane 19:1. Colorless crystals were obtained from pentane at $-20^{\circ} \mathrm{C}$. Those of $16 \mathbf{c}$ could be isolated by fractional recrystallization of the remaining mixture from pentane at $-80^{\circ} \mathrm{C}$ followed by recrystallization at $-20^{\circ} \mathrm{C}$. NMR data of the syn isomer $16 \mathbf{d}$ could be obtained from the residual mixture of isomers.

syn-(1S,3S,4S)- and (1R,3R,4R)-Isomer 16a: ${ }^{31} \mathrm{P}$ NMR $\quad(101.25 \mathrm{MHz}$, $\left.\mathrm{CDCl}_{3}\right): \delta=-142.5 \mathrm{ppm}\left({ }^{1} J(\mathrm{P}, \mathrm{W})=257.8 \mathrm{~Hz}\right)$.

anti-(1R,3S,4R)- and (1S,3R,4S)-Isomer 16b: m.p. $47-48{ }^{\circ} \mathrm{C} ;{ }^{1} \mathrm{H} N M R$ $\left(400.13 \mathrm{MHz}, \mathrm{CDCl}_{3}\right): \delta=1.08-1.11\left(\mathrm{dddd},{ }^{2} J(\mathrm{H}, \mathrm{H})=8.4,{ }^{4} J(\mathrm{H}, \mathrm{H})=2.4\right.$, $\left.{ }^{4} J(\mathrm{H}, \mathrm{H})=1.8,{ }^{4} J(\mathrm{H}, \mathrm{H})=0.7 \mathrm{~Hz}, 1 \mathrm{H} ; \mathrm{CH}\right), 1.50\left(\mathrm{ddd},{ }^{2} J(\mathrm{H}, \mathrm{H})=8.4,{ }^{4} J-\right.$ $\left.(\mathrm{H}, \mathrm{H})=2.4,{ }^{4} J(\mathrm{H}, \mathrm{H})=1.8 \mathrm{~Hz}, 1 \mathrm{H} ; \mathrm{CH}\right), 1.61\left(\mathrm{ddd},{ }^{2} J(\mathrm{H}, \mathrm{H})=4.2,{ }^{3} J-\right.$ $\left.(\mathrm{H}, \mathrm{P})=6.8,{ }^{4} J(\mathrm{H}, \mathrm{H})=0.7 \mathrm{~Hz}, 1 \mathrm{H} ; \mathrm{PCC} H\right), 1.83\left(\mathrm{dd},{ }^{2} J(\mathrm{H}, \mathrm{H})=7.7,{ }^{2} J-\right.$ $(\mathrm{H}, \mathrm{P})=1.2 \mathrm{~Hz}, 1 \mathrm{H} ; \mathrm{PCH}), 2.02\left(\mathrm{dd},{ }^{2} J(\mathrm{H}, \mathrm{H})=7.7,{ }^{2} J(\mathrm{H}, \mathrm{P})=7.4 \mathrm{~Hz}, 1 \mathrm{H}\right.$; $\mathrm{PCH}), 2.11\left(\mathrm{dd},{ }^{2} J(\mathrm{H}, \mathrm{H})=4.2,{ }^{3} J(\mathrm{H}, \mathrm{P})=9.9 \mathrm{~Hz}, 1 \mathrm{H} ; \mathrm{PCCH}\right), 5.36\left(\mathrm{t},{ }^{4} J-\right.$ $(\mathrm{H}, \mathrm{H})=2.4 \mathrm{~Hz}, 1 \mathrm{H} ;=\mathrm{CH}), 5.39\left(\mathrm{t},{ }^{4} J(\mathrm{H}, \mathrm{H})=1.8 \mathrm{~Hz}, 1 \mathrm{H} ;=\mathrm{CH}\right), 7.28-$ $7.41 \mathrm{ppm}(\mathrm{m}, 5 \mathrm{H} ; \mathrm{Ph} H) ;{ }^{13} \mathrm{C}\left\{{ }^{1} \mathrm{H}\right\}$ NMR $\left(100.62 \mathrm{MHz}, \mathrm{CDCl}_{3}\right): \delta=9.5(\mathrm{~d}$, $\left.{ }^{3} J(\mathrm{C}, \mathrm{P})=4.6 \mathrm{~Hz} ; \mathrm{CH}_{2}\right), 17.8\left(\mathrm{~d},{ }^{1} J(\mathrm{C}, \mathrm{P})=5.8 \mathrm{~Hz} ; \mathrm{PCH}_{2}\right), 19.5\left(\mathrm{~s} ; \mathrm{PCCH}_{2}\right)$, $19.5(\mathrm{~s} ; \mathrm{PCC}), 29.0\left(\mathrm{~d},{ }^{1} J(\mathrm{C}, \mathrm{P})=7.7 \mathrm{~Hz} ; \mathrm{PC}\right), 101.6\left(\mathrm{~s} ;=\mathrm{CH}_{2}\right), 128.6\left(\mathrm{~d},{ }^{3} J-\right.$ $(\mathrm{C}, \mathrm{P})=10.9 \mathrm{~Hz} ; m-\mathrm{Ph}), 130.5\left(\mathrm{~d},{ }^{4} J(\mathrm{C}, \mathrm{P})=2.5 \mathrm{~Hz} ; p-\mathrm{Ph}\right), 131.3\left(\mathrm{~d},{ }^{1} J-\right.$ $(\mathrm{C}, \mathrm{P})=25.8 \mathrm{~Hz}$; ipso-Ph), $131.6\left(\mathrm{~d},{ }^{2} J(\mathrm{C}, \mathrm{P})=14.0 \mathrm{~Hz} ; o-\mathrm{Ph}\right), 133.7(\mathrm{~s} ;=$ C), $195.5\left(\mathrm{~d},{ }^{2} J(\mathrm{C}, \mathrm{P})=8.3,{ }^{1} J(\mathrm{C}, \mathrm{W})=125.5 \mathrm{~Hz} ;\right.$ cis-CO $), 197.6 \mathrm{ppm}\left(\mathrm{d},{ }^{2} J-\right.$ $(\mathrm{C}, \mathrm{P})=30.4 \mathrm{~Hz}$; trans $-\mathrm{CO}) ;{ }^{31} \mathrm{P}$ NMR $\left(101.25 \mathrm{MHz}, \mathrm{CDCl}_{3}\right): \delta=-144.0$ $\left({ }^{1} J(\mathrm{P}, \mathrm{W})=258.2 \mathrm{~Hz}\right) ; \mathrm{IR}(\mathrm{KBr}): \tilde{v}=1925\left(\mathrm{~s} / \mathrm{br}, \mathrm{CO}_{\mathrm{eq}}\right), 2074 \mathrm{~cm}^{-1}(\mathrm{~m}$, $\left.\mathrm{CO}_{\mathrm{ax}}\right)$; MS $(70 \mathrm{eV}): m / z(\%): 524(4)[M]^{+}, 468(8)[M-2 \mathrm{CO}]^{+}, 440(3)$ $[M-3 \mathrm{CO}]^{+}, 412(12)[M-4 \mathrm{CO}]^{+}, 384(52)[M-5 \mathrm{CO}]^{+}$; HR-MS (EI): $m / z$ : calcd for $\mathrm{C}_{18} \mathrm{H}_{13} \mathrm{O}_{5} \mathrm{P}^{184} \mathrm{~W}$ : 524.00104; found: 524.00082 .

anti-(1R,3S,4S)- and $(1 S, 3 R, 4 R)-$ Isomer $16 \mathrm{c}:{ }^{1} \mathrm{H} N M R \quad(400.13 \mathrm{MHz}$, $\left.\mathrm{CDCl}_{3}\right): \delta=1.45-1.54(\mathrm{~m}, 2 \mathrm{H}, \mathrm{CH}), 1.70\left(\mathrm{dd},{ }^{2} J(\mathrm{H}, \mathrm{H})=7.7,{ }^{2} J(\mathrm{H}, \mathrm{P})=\right.$ $0.8 \mathrm{~Hz}, 1 \mathrm{H} ; \mathrm{PCH}), 1.85\left(\mathrm{dd},{ }^{2} J(\mathrm{H}, \mathrm{H})=4.2,{ }^{3} J(\mathrm{H}, \mathrm{P})=10.8 \mathrm{~Hz}, 1 \mathrm{H}\right.$; $\mathrm{PCCH}), 1.92\left(\mathrm{dd},{ }^{2} J(\mathrm{H}, \mathrm{H})=4.2,{ }^{3} J(\mathrm{H}, \mathrm{P})=2.1 \mathrm{~Hz}, 1 \mathrm{H} ; \mathrm{PCC} H\right), 2.13(\mathrm{dd}$, $\left.{ }^{2} J(\mathrm{H}, \mathrm{H})=7.7,{ }^{2} J(\mathrm{H}, \mathrm{P})=7.0 \mathrm{~Hz}, 1 \mathrm{H} ; \mathrm{PCH}\right), 5.51-5.53\left(\mathrm{~m}, 2 \mathrm{H} ;=\mathrm{CH}_{2}\right)$, 7.40-7.45 (m, 3H; $m-\mathrm{Ph} H, p-\mathrm{Ph} H), 7.51-7.57 \mathrm{ppm}(\mathrm{m}, 2 \mathrm{H} ; o-\mathrm{Ph} H)$; ${ }^{13} \mathrm{C}\left\{{ }^{1} \mathrm{H}\right\}$ NMR $\left(100.62 \mathrm{MHz}, \mathrm{CDCl}_{3}\right): \delta=9.4\left(\mathrm{~d},{ }^{3} J(\mathrm{C}, \mathrm{P})=2.3 \mathrm{~Hz} ; \mathrm{CH}_{2}\right)$, $17.5\left(\mathrm{~d},{ }^{1} J(\mathrm{C}, \mathrm{P})=6.1 \mathrm{~Hz} ; \mathrm{PCH}_{2}\right), 19.3\left(\mathrm{~d},{ }^{2} J(\mathrm{C}, \mathrm{P})=3.5 \mathrm{~Hz} ; \mathrm{PCCH}_{2}\right), 20.5$ (s; PCC), $29.1\left(\mathrm{~d},{ }^{1} J(\mathrm{C}, \mathrm{P})=7.5 \mathrm{~Hz} ; \mathrm{PC}\right), 102.7\left(\mathrm{~s} ;=\mathrm{CH}_{2}\right), 128.8\left(\mathrm{~d},{ }^{3} \mathrm{~J}-\right.$ $(\mathrm{C}, \mathrm{P})=10.5 \mathrm{~Hz} ; m-\mathrm{Ph}), 130.4\left(\mathrm{~d},{ }^{4} J(\mathrm{C}, \mathrm{P})=2.2 \mathrm{~Hz} ; p-\mathrm{Ph}\right), 131.4\left(\mathrm{~d},{ }^{2} J-\right.$ $(\mathrm{C}, \mathrm{P})=13.3 \mathrm{~Hz} ; o-\mathrm{Ph}), 132.9\left(\mathrm{~d},{ }^{3} J(\mathrm{C}, \mathrm{P})=3.6 \mathrm{~Hz} ;=C\right), 134.2\left(\mathrm{~d},{ }^{1} J(\mathrm{C}, \mathrm{P})=\right.$ $25.3 \mathrm{~Hz}$; ipso-Ph), $195.6\left(\mathrm{~d},{ }^{2} J(\mathrm{C}, \mathrm{P})=8.4,{ }^{1} J(\mathrm{C}, \mathrm{W})=125.6 \mathrm{~Hz}\right.$; cis-CO $)$, $197.7 \mathrm{ppm} \quad\left(\mathrm{d},{ }^{2} J(\mathrm{C}, \mathrm{P})=30.7 \mathrm{~Hz} ;\right.$ trans $\left.-\mathrm{CO}\right) ;{ }^{31} \mathrm{P} \mathrm{NMR} \quad(101.25 \mathrm{MHz}$, $\left.\mathrm{CDCl}_{3}\right): \delta=-148.3 \mathrm{ppm}\left({ }^{1} J(\mathrm{P}, \mathrm{W})=257.2 \mathrm{~Hz}\right)$.

syn-(1S,3S,4R)- and $(1 \boldsymbol{R}, 3 \boldsymbol{R}, 4 S)$-Isomer $16 \mathrm{~d}$ : ${ }^{1} \mathrm{H}$ NMR $(400.13 \mathrm{MHz}$, $\left.\mathrm{CDCl}_{3}\right): \delta=1.65\left(\mathrm{~d},{ }^{2} J(\mathrm{H}, \mathrm{H})=7.5 \mathrm{~Hz}, 1 \mathrm{H} ; \mathrm{PCH}\right), 1.71-1.74(\mathrm{~m}, 1 \mathrm{H}$; $\mathrm{CH}), 1.78-1.82(\mathrm{~m}, 2 \mathrm{H} ; \mathrm{PCCH}, \mathrm{CH}), 1.99-2.07\left(\mathrm{~m}, 1 \mathrm{H},{ }^{3} \mathrm{~J}(\mathrm{H}, \mathrm{P})=4.1 \mathrm{~Hz}\right.$, $\mathrm{PCCH}), 2.05\left(\mathrm{~d},{ }^{2} J(\mathrm{H}, \mathrm{H})=7.5,{ }^{2} J(\mathrm{H}, \mathrm{P})=\sim 7 \mathrm{~Hz}, 1 \mathrm{H} ; \mathrm{PCH}\right), 5.29\left(\mathrm{t},{ }^{4} J-\right.$ $(\mathrm{H}, \mathrm{H})=2.4 \mathrm{~Hz}, 1 \mathrm{H} ;=\mathrm{CH}), 5.40\left(\mathrm{t},{ }^{4} J(\mathrm{H}, \mathrm{H})=1.7 \mathrm{~Hz}, 1 \mathrm{H} ;=\mathrm{CH}\right), 7.41-$ $\left.7.45(\mathrm{~m}, 3 \mathrm{H} ; m-\mathrm{Ph} H, p-\mathrm{Ph} H), 7.52-7.58 \mathrm{ppm}(\mathrm{m}, 2 \mathrm{H} ; o-\mathrm{Ph} H) ;{ }^{13} \mathrm{C}_{1}{ }^{1} \mathrm{H}\right\}$ NMR $\left(100.62 \mathrm{MHz}, \mathrm{CDCl}_{3}\right): \delta=10.5\left(\mathrm{~d},{ }^{3} J(\mathrm{C}, \mathrm{P})=4.3 \mathrm{~Hz} ; \mathrm{CH}_{2}\right), 17.9(\mathrm{~d}$, $\left.{ }^{1} J(\mathrm{C}, \mathrm{P})=6.0 \mathrm{~Hz} ; \mathrm{PCH}_{2}\right), 18.9\left(\mathrm{~d},{ }^{2} J(\mathrm{C}, \mathrm{P})=3.8 \mathrm{~Hz} ; \mathrm{PCCH}_{2}\right), 20.4 \quad(\mathrm{~s} ;$ PCC), $28.3\left(\mathrm{~d},{ }^{1} J(\mathrm{C}, \mathrm{P})=9.3 \mathrm{~Hz} ; \mathrm{PC}\right), 101.4\left(\mathrm{~d},{ }^{4} J(\mathrm{C}, \mathrm{P})=1.4 \mathrm{~Hz} ;=\mathrm{CH}_{2}\right)$, $128.8\left(\mathrm{~d},{ }^{3} J(\mathrm{C}, \mathrm{P})=10.4 \mathrm{~Hz} ; m-\mathrm{Ph}\right), 130.4\left(\mathrm{~d},{ }^{4} J(\mathrm{C}, \mathrm{P})=2.4 \mathrm{~Hz} ; p-\mathrm{Ph}\right), 131.3$ $\left(\mathrm{d},{ }^{2} J(\mathrm{C}, \mathrm{P})=13.0 \mathrm{~Hz} ; o-\mathrm{Ph}\right), 132.7\left(\mathrm{~d},{ }^{3} J(\mathrm{C}, \mathrm{P})=2.2 \mathrm{~Hz} ;=C\right), 134.4\left(\mathrm{~d},{ }^{1} J-\right.$ $(\mathrm{C}, \mathrm{P})=25.6 \mathrm{~Hz} ;$ ipso-Ph $), 195.5\left(\mathrm{~d},{ }^{2} \mathrm{~J}(\mathrm{C}, \mathrm{P})=8.3,{ }^{1} \mathrm{~J}(\mathrm{C}, \mathrm{W})=125.4 \mathrm{~Hz}\right.$; cis-
CO), $197.6 \mathrm{ppm}\left(\mathrm{d},{ }^{2} \mathrm{~J}(\mathrm{C}, \mathrm{P})=30.4 \mathrm{~Hz}\right.$; trans-CO $) ;{ }^{31} \mathrm{P}$ NMR $(101.25 \mathrm{MHz}$, $\left.\mathrm{CDCl}_{3}\right): \delta=-148.5 \mathrm{ppm}\left({ }^{1} J(\mathrm{P}, \mathrm{W})=256.2 \mathrm{~Hz}\right)$

\section{1,6-Di(pentacarbonyltungstino)-1,6-diphenyl-1,6-diphosphadispiro-}

[2.0.0.2.1.1]nonane (17): Compound 16b $(88 \mathrm{mg}, 0.17 \mathrm{mmol})$, complex 8 $(219 \mathrm{mg}, 0.33 \mathrm{mmol})$ and $\mathrm{CuCl}(10 \mathrm{mg}, 0.1 \mathrm{mmol})$ were heated in toluene $(1 \mathrm{~mL})$ at $55^{\circ} \mathrm{C}$ for $3 \mathrm{~h}$. Evaporation to dryness and chromatography of the residue over silica gel eluting with pentane/dichloromethane 9:1 gave 17a and $\mathbf{b}$ in a $10: 7$ ratio $(127 \mathrm{mg}, 78 \%)$ as a colorless solid. Fractional recrystallization from pentane $/ \mathrm{CH}_{2} \mathrm{Cl}_{2}$ at $0{ }^{\circ} \mathrm{C}$ afforded colorless crystals of $\mathbf{1 7} \mathbf{a}$

anti,anti-17a: m.p. $198^{\circ} \mathrm{C}(\mathrm{decomp}) ;{ }^{1} \mathrm{H} \mathrm{NMR}\left(400.13 \mathrm{MHz}, \mathrm{CDCl}_{3}\right): \delta=$ $0.95\left(\mathrm{dd},{ }^{3} \mathrm{~J}(\mathrm{H}, \mathrm{P})=6.5,{ }^{2} \mathrm{~J}(\mathrm{H}, \mathrm{H})=4.9 \mathrm{~Hz}, 2 \mathrm{H} ; \mathrm{PCCH}\right), 1.59\left(\mathrm{~d},{ }^{2} \mathrm{~J}(\mathrm{H}, \mathrm{H})=\right.$ $7.7 \mathrm{~Hz}, 2 \mathrm{H} ; \mathrm{PCH}), 1.76\left(\mathrm{dd},{ }^{3} J(\mathrm{H}, \mathrm{P})=9.9,{ }^{2} J(\mathrm{H}, \mathrm{H})=4.9 \mathrm{~Hz}, 2 \mathrm{H} ; \mathrm{PCCH}\right)$, $2.24\left(\mathrm{dd},{ }^{2} J(\mathrm{H}, \mathrm{P})=7.1,{ }^{2} J(\mathrm{H}, \mathrm{H})=7.7 \mathrm{~Hz}, 2 \mathrm{H} ; \mathrm{PCH}\right), 7.40-7.46 \mathrm{ppm}(\mathrm{m}$, $10 \mathrm{H} ; \mathrm{Ph} H) ;{ }^{13} \mathrm{C}\left\{{ }^{1} \mathrm{H}\right\}$ NMR $\left(100.62 \mathrm{MHz}, \mathrm{CDCl}_{3}\right): \delta=15.1\left(\mathrm{~m},{ }^{(2+3)} \mathrm{J}-\right.$ $(\mathrm{C}, \mathrm{P})=4.6 \mathrm{~Hz} ; \mathrm{PCCH}), 15.3\left(\mathrm{~d},{ }^{(1+4)} J(\mathrm{C}, \mathrm{P})=6.3 \mathrm{~Hz} ; \mathrm{PCH}_{2}\right), 25.4\left(\mathrm{t},{ }^{2} J-\right.$ $(\mathrm{C}, \mathrm{P})=5.5 \mathrm{~Hz} ; \mathrm{PCC}), 26.3\left(\mathrm{~d},{ }^{(1+3} J(\mathrm{C}, \mathrm{P})=8.8 \mathrm{~Hz} ; \mathrm{PC}\right), 128.8\left(\mathrm{~d},{ }^{3} J-\right.$ $(\mathrm{C}, \mathrm{P})=10.8 \mathrm{~Hz} ; m-\mathrm{Ph}), 130.8\left(\mathrm{~m},{ }^{4} J(\mathrm{C}, \mathrm{P})=2.3 \mathrm{~Hz} ; p-\mathrm{Ph}\right), 131.4\left(\mathrm{~d},{ }^{2} J-\right.$ $(\mathrm{C}, \mathrm{P})=13.9 \mathrm{~Hz} ; o-\mathrm{Ph}), 131.5\left(\mathrm{~d},{ }^{1} J(\mathrm{C}, \mathrm{P})=26.3 \mathrm{~Hz} ;\right.$ ipso-Ph $), 195.3\left(\mathrm{~d},{ }^{2} J-\right.$ $(\mathrm{C}, \mathrm{P})=8.4,{ }^{1} J(\mathrm{C}, \mathrm{W})=125.4 \mathrm{~Hz} ;$ cis-CO $), 197.3 \mathrm{ppm}\left(\mathrm{d},{ }^{2} J(\mathrm{C}, \mathrm{P})=31.1 \mathrm{~Hz}\right.$; trans-CO); ${ }^{31} \mathrm{P}$ NMR $\quad\left(101.25 \mathrm{MHz}, \quad \mathrm{CDCl}_{3}\right): \delta=-147.9 \quad\left({ }^{1} \mathrm{~J}(\mathrm{P}, \mathrm{W})=\right.$ $261.2 \mathrm{~Hz})$; IR ( $\mathrm{KBr}): \tilde{v}=1917$ and $1935\left(\mathrm{~s} / \mathrm{br}, \mathrm{CO}_{\mathrm{eq}}\right), 1991$ (w, CO $\left.\mathrm{eq}\right)$, $2074 \mathrm{~cm}^{-1}\left(\mathrm{~m}, \mathrm{CO}_{\mathrm{ax}}\right) ; \mathrm{MS}(70 \mathrm{eV}): \mathrm{m} / z$ (\%): $956(8)[M]^{+}, 900$ (1) $[M-2 \mathrm{CO}]^{+}, 844(16)[M-4 \mathrm{CO}]^{+}, 788$ (20) $[M-6 \mathrm{CO}]^{+}, 732(40)$ $[M-8 \mathrm{CO}]^{+}, 676(100)[M-10 \mathrm{CO}]^{+}$; HR-MS (EI): $\mathrm{m} / z$ : calcd for $\mathrm{C}_{29} \mathrm{H}_{18} \mathrm{O}_{10} \mathrm{P}_{2}{ }^{184} \mathrm{~W}_{2}$ : 955.93945; found: 955.93875 ; elemental analysis calcd (\%) for $\mathrm{C}_{29} \mathrm{H}_{18} \mathrm{O}_{10} \mathrm{P}_{2}^{184} \mathrm{~W}_{2}$ : C 36.43, H 1.90; found: C 36.12, H 1.95.

anti,syn-17b: ${ }^{1} \mathrm{H}$ NMR $\left(400.13 \mathrm{MHz}, \mathrm{CDCl}_{3}\right): \delta=1.05\left(\mathrm{dd},{ }^{3} J(\mathrm{H}, \mathrm{P})=1.5\right.$, $\left.{ }^{2} J(\mathrm{H}, \mathrm{H})=4.6 \mathrm{~Hz}, 1 \mathrm{H} ; \mathrm{PCC} H\right), 1.58\left(\mathrm{dd},{ }^{3} J(\mathrm{H}, \mathrm{P})=10.8,{ }^{2} J(\mathrm{H}, \mathrm{H})=4.7 \mathrm{~Hz}\right.$, $1 \mathrm{H} ; \mathrm{PCC} H), 1.75\left(\mathrm{~d},{ }^{2} J(\mathrm{H}, \mathrm{H})=8.1 \mathrm{~Hz}, 1 \mathrm{H} ; \mathrm{PCH}\right), 1.80\left(\mathrm{dd},{ }^{3} J(\mathrm{H}, \mathrm{P})=\right.$ $\left.6.5,{ }^{2} J(\mathrm{H}, \mathrm{H})=5.0 \mathrm{~Hz}, 1 \mathrm{H} ; \mathrm{PCCH}\right), 1.81-1.87(\mathrm{~m}, 2 \mathrm{H} ; \mathrm{PCH}), 2.00\left(\mathrm{dd},{ }^{3} \mathrm{~J}-\right.$ $\left.(\mathrm{H}, \mathrm{P})=9.6,{ }^{2} J(\mathrm{H}, \mathrm{H})=5.0 \mathrm{~Hz}, 1 \mathrm{H} ; \mathrm{PCCH}\right), 2.28\left(\mathrm{dd},{ }^{2} J(\mathrm{H}, \mathrm{P})=6.9,{ }^{2} J-\right.$ $(\mathrm{H}, \mathrm{H})=8.2 \mathrm{~Hz}, 1 \mathrm{H} ; \mathrm{PCH}), 7.38-7.48 \mathrm{ppm}(\mathrm{m}, 10 \mathrm{H} ; \mathrm{Ph} H) ;{ }^{13} \mathrm{C}\left\{{ }^{1} \mathrm{H}\right\}$ NMR $\left(100.62 \mathrm{MHz}, \mathrm{CDCl}_{3}\right): \delta=14.2\left(\mathrm{dd},{ }^{2} J(\mathrm{C}, \mathrm{P})=3.9 \mathrm{~Hz} ; \mathrm{PCCH}\right), 15.5\left(\mathrm{~d},{ }^{1} J-\right.$ $\left.(\mathrm{C}, \mathrm{P})=6.1 \mathrm{~Hz} ; \mathrm{PCH}_{2}\right), 16.0\left(\mathrm{~d},{ }^{1} J(\mathrm{C}, \mathrm{P})=6.3 \mathrm{~Hz} ; \mathrm{PCH}_{2}\right), 16.1\left(\mathrm{~d},{ }^{2} J(\mathrm{C}, \mathrm{P})=\right.$ $3.1 \mathrm{~Hz} ; \mathrm{PCCH}), 25.6\left(\mathrm{~d},{ }^{1} J(\mathrm{C}, \mathrm{P})=9.8 \mathrm{~Hz} ; \mathrm{PC}\right), 26.1\left(\mathrm{~d},{ }^{1} J(\mathrm{C}, \mathrm{P})=5.5 \mathrm{~Hz}\right.$; $\mathrm{PC}), 26.4\left(\mathrm{dd},{ }^{2} J(\mathrm{C}, \mathrm{P})=2.1,{ }^{2} J(\mathrm{C}, \mathrm{P})=8.9 \mathrm{~Hz} ; \mathrm{PCC}\right), 128.7\left(\mathrm{~d},{ }^{3} J(\mathrm{C}, \mathrm{P})=\right.$ $10.5 \mathrm{~Hz} ; m-\mathrm{Ph}), 128.9\left(\mathrm{~d},{ }^{3} J(\mathrm{C}, \mathrm{P})=10.4 \mathrm{~Hz} ; m-\mathrm{Ph}\right), 130.5\left(\mathrm{~d},{ }^{4} J(\mathrm{C}, \mathrm{P})=\right.$ $2.3 \mathrm{~Hz} ; p-\mathrm{Ph}), 130.6\left(\mathrm{~d},{ }^{4} J(\mathrm{C}, \mathrm{P})=2.4 \mathrm{~Hz} ; p-\mathrm{Ph}\right), 131.2\left(\mathrm{~d},{ }^{2} J(\mathrm{C}, \mathrm{P})=\right.$ $13.0 \mathrm{~Hz} ; o-\mathrm{Ph}), 131.4\left(\mathrm{~d},{ }^{2} J(\mathrm{C}, \mathrm{P})=13.4 \mathrm{~Hz} ; o-\mathrm{Ph}\right), 131.7\left(\mathrm{~d},{ }^{1} J(\mathrm{C}, \mathrm{P})=\right.$ $25.4 \mathrm{~Hz}$; ipso-Ph), $133.8\left(\mathrm{~d},{ }^{1} J(\mathrm{C}, \mathrm{P})=26.1 \mathrm{~Hz} ;\right.$ ipso-Ph $), 195.3\left(\mathrm{~d},{ }^{2} J-\right.$ $(\mathrm{C}, \mathrm{P})=8.1,{ }^{1} J(\mathrm{C}, \mathrm{W})=125.4 \mathrm{~Hz} ;$ cis-CO $), 195.5 \quad\left(\mathrm{~d},{ }^{2} J(\mathrm{C}, \mathrm{P})=8.0,{ }^{1} J-\right.$ $(\mathrm{C}, \mathrm{W})=125.4 \mathrm{~Hz} ; \quad$ cis-CO $), 197.3 \quad\left(\mathrm{~d},{ }^{2} J(\mathrm{C}, \mathrm{P})=30.4 \mathrm{~Hz} ;\right.$ trans $\left.-\mathrm{CO}\right)$, $197.4 \mathrm{ppm} \quad\left(\mathrm{d},{ }^{2} J(\mathrm{C}, \mathrm{P})=30.6 \mathrm{~Hz}\right.$; trans-CO); ${ }^{31} \mathrm{P} \mathrm{NMR} \quad(101.25 \mathrm{MHz}$, $\left.\mathrm{CDCl}_{3}\right): \delta=-150.4\left({ }^{1} J(\mathrm{P}, \mathrm{W})=260.2,{ }^{4} J(\mathrm{P}, \mathrm{P})=5.1 \mathrm{~Hz}\right),-150.5 \mathrm{ppm}\left({ }^{1} J-\right.$ $\left.(\mathrm{P}, \mathrm{W})=258.2,{ }^{4} J(\mathrm{P}, \mathrm{P})=4.1 \mathrm{~Hz}\right)$.

Synthesis of 7 and 19: Complex $8(0.65 \mathrm{~g}, 1.00 \mathrm{mmol})$, perspirocyclopropanated bicyclopropylidene $\mathbf{1 8}(0.22 \mathrm{~g}, 1.20 \mathrm{mmol})$ and $\mathrm{CuCl}(10 \mathrm{mg}$, $0.1 \mathrm{mmol}$ ) were stirred at room temperature for $20 \mathrm{~h}$ in toluene $(4 \mathrm{~mL})$. Evaporation to dryness and chromatography of the residue over silica gel eluting with pentane/dichloromethane 19:1 yielded both $7(0.26 \mathrm{~g}, 42 \%)$ and $19(40 \mathrm{mg}, 6 \%)$ as pale yellow solids. Recrystallization of 7 from hexane at $-20^{\circ} \mathrm{C}$ and 19 from diethyl ether at $-5^{\circ} \mathrm{C}$ afforded colorless crystals for both.

(15-Phenyl-15-phosphahexaspiro[2.0.2.0.0.0.2.0.2.0.1]pentadec-15-yl)pentacarbonyltungsten (7): m.p. $178-179^{\circ} \mathrm{C}$ (decomp); ${ }^{1} \mathrm{H}$ NMR $(400.1 \mathrm{MHz}$, $\left.\mathrm{CDCl}_{3}\right): \delta=0.60-0.68\left(\mathrm{~m}, 4 \mathrm{H} ; \mathrm{CH}_{2}\right), 0.75-0.80\left(\mathrm{~m}, 2 \mathrm{H} ; \mathrm{CH}_{2}\right), 0.85-0.96$ $\left(\mathrm{m}, 6 \mathrm{H} ; \mathrm{CH}_{2}\right), 0.96-1.02\left(\mathrm{~m}, 2 \mathrm{H} ; \mathrm{CH}_{2}\right), 1.22-1.28\left(\mathrm{~m}, 2 \mathrm{H} ; \mathrm{CH}_{2}\right), 7.25-$ $7.39 \mathrm{ppm}(\mathrm{m}, 5 \mathrm{H} ; \mathrm{Ph} H) ;{ }^{13} \mathrm{C}\left\{{ }^{1} \mathrm{H}\right\}$ NMR $\left(100.6 \mathrm{MHz}, \mathrm{CDCl}_{3}\right): \delta=4.4(\mathrm{~d}$, $\left.{ }^{3} J(\mathrm{C}, \mathrm{P})=4.0 \mathrm{~Hz} ; \mathrm{CH}_{2}\right), 4.7\left(\mathrm{~d},{ }^{3} J(\mathrm{C}, \mathrm{P})=3.3 \mathrm{~Hz} ; \mathrm{CH}_{2}\right), 7.1\left(\mathrm{~d},{ }^{3} J(\mathrm{C}, \mathrm{P})=\right.$ $\left.2.1 \mathrm{~Hz} ; \mathrm{CH}_{2}\right), 7.4\left(\mathrm{~s} ; \mathrm{CH}_{2}\right), 21.8\left(\mathrm{~d},{ }^{2} J(\mathrm{C}, \mathrm{P})=5.3 \mathrm{~Hz} ; \mathrm{PCC}\right), 21.9\left(\mathrm{~d},{ }^{2} J-\right.$ $(\mathrm{C}, \mathrm{P})=1.2 \mathrm{~Hz} ; \mathrm{PCC}), 34.5\left(\mathrm{~d},{ }^{1} J(\mathrm{C}, \mathrm{P})=6.2 \mathrm{~Hz} ; \mathrm{PC}\right), 128.2\left(\mathrm{~d},{ }^{3} J(\mathrm{C}, \mathrm{P})=\right.$ $10.2 \mathrm{~Hz} ; m-\mathrm{Ph}), 130.0\left(\mathrm{~d},{ }^{4} J(\mathrm{C}, \mathrm{P})=2.6 \mathrm{~Hz} ; p-\mathrm{Ph}\right), 131.9\left(\mathrm{~d},{ }^{2} J(\mathrm{C}, \mathrm{P})=\right.$ $13.1 \mathrm{~Hz} ; o-\mathrm{Ph}), 132.9$ (d, ${ }^{1} J(\mathrm{C}, \mathrm{P})=19.9 \mathrm{~Hz} ;$ ipso-Ph), $195.9\left(\mathrm{~d},{ }^{2} J(\mathrm{C}, \mathrm{P})=\right.$ $8.3,{ }^{1} J(\mathrm{C}, \mathrm{W})=125.4 \mathrm{~Hz}$; cis-CO $), 198.9 \mathrm{ppm}\left(\mathrm{d},{ }^{2} J(\mathrm{C}, \mathrm{P})=29.5 \mathrm{~Hz}\right.$; trans$\mathrm{CO}) ;{ }^{31} \mathrm{P}\left\{{ }^{1} \mathrm{H}\right\}$ NMR $\left(101.3 \mathrm{MHz}, \mathrm{CDCl}_{3}\right): \delta=-119.6 \mathrm{ppm} \quad\left({ }^{1} \mathrm{~J}(\mathrm{P}, \mathrm{W})=\right.$ $250.1 \mathrm{~Hz})$; IR (KBr): $\tilde{v}=1923,1942\left(\mathrm{~s} / \mathrm{br}, \mathrm{CO}_{\mathrm{eq}}\right), 1985$ (w, $\left.\mathrm{CO}_{\mathrm{eq}}\right)$, 
$2072 \mathrm{~cm}^{-1}\left(\mathrm{~m}, \mathrm{CO}_{\mathrm{ax}}\right)$; MS (70 eV): $m / z(\%): 616$ (4) $[M]^{+}$; HR-MS (EI, $70 \mathrm{eV}$ ): $\mathrm{m} / z$ : calcd for $\mathrm{C}_{25} \mathrm{H}_{21} \mathrm{O}_{5} \mathrm{P}^{184} \mathrm{~W}$ : 616.06366 ; found: 616.06655 .

\{2-Phenyl-2-phospha-3:3,4:4,6:6,7:7-tetrakisethanobicyclo[3.2.0]hept-1(5)en-2-yljpentacarbonyltungsten $\quad$ (19): m.p. $\quad 146-148^{\circ} \mathrm{C}$; ${ }^{1} \mathrm{H}$ NMR $\left(400.1 \mathrm{MHz}, \mathrm{CDCl}_{3}\right): \delta=0.23-0.29\left(\mathrm{~m}, 1 \mathrm{H} ; \mathrm{CH}_{2}\right), 0.33-0.42(\mathrm{~m}, 2 \mathrm{H}$; $\left.\mathrm{CH}_{2}\right), 0.51-0.59\left(\mathrm{~m}, 2 \mathrm{H} ; \mathrm{CH}_{2}\right), 0.61-0.71\left(\mathrm{~m}, 6 \mathrm{H} ; \mathrm{CH}_{2}\right), 0.79-0.83(\mathrm{~m}$, $\left.2 \mathrm{H}, \mathrm{CH}_{2}\right)$ 0.91-0.98 (m, 2H; $\left.\mathrm{CH}_{2}\right), 1.10-1.14\left(\mathrm{~m}, 1 \mathrm{H}, \mathrm{CH}_{2}\right), 7.40-7.44(\mathrm{~m}$, $3 \mathrm{H} ; m-\mathrm{Ph} H, p-\mathrm{Ph} H), 7.50-7.56 \mathrm{ppm}(\mathrm{m}, 2 \mathrm{H} ; o-\mathrm{Ph} H) ;{ }^{13} \mathrm{C}\left\{{ }^{1} \mathrm{H}\right\} \quad \mathrm{NMR}$ $\left(100.6 \mathrm{MHz}, \mathrm{CDCl}_{3}\right): \delta=6.3,6.6,7.7,7.8,7.9,8.8\left(\mathrm{~s} ; \mathrm{CH}_{2}\right), 10.1\left(\mathrm{~d},{ }^{2} J-\right.$ $\left.(\mathrm{C}, \mathrm{P})=2.9 \mathrm{~Hz} ; \mathrm{PCCH}_{2}\right), 10.4\left(\mathrm{~d},{ }^{2} J(\mathrm{C}, \mathrm{P})=7.1 \mathrm{~Hz} ; \mathrm{PCCH}_{2}\right), 30.6\left(\mathrm{~d},{ }^{2} J-\right.$ $(\mathrm{C}, \mathrm{P})=7.7 \mathrm{~Hz} ; \mathrm{PCC}), 34.9\left(\mathrm{~d},{ }^{1} J(\mathrm{C}, \mathrm{P})=39.7 \mathrm{~Hz} ; \mathrm{PC}\right), 35.2\left(\mathrm{~d},{ }^{2} J(\mathrm{C}, \mathrm{P})=\right.$ $22.1 \mathrm{~Hz} ; \mathrm{P}(\mathrm{C}=) C), 35.8\left(\mathrm{~d},{ }^{3} J(\mathrm{C}, \mathrm{P})=4.2 \mathrm{~Hz} ; \mathrm{PC}=\mathrm{CC}\right), 128.5\left(\mathrm{~d},{ }^{3} J(\mathrm{C}, \mathrm{P})=\right.$ $9.6 \mathrm{~Hz} ; m-\mathrm{Ph}), 130.3\left(\mathrm{~d},{ }^{4} J(\mathrm{C}, \mathrm{P})=2.1 \mathrm{~Hz} ; p-\mathrm{Ph}\right), 131.4\left(\mathrm{~d},{ }^{2} J(\mathrm{C}, \mathrm{P})=\right.$ $12.3 \mathrm{~Hz} ; o-\mathrm{Ph}), 136.3\left(\mathrm{~d},{ }^{1} J(\mathrm{C}, \mathrm{P})=32.5 \mathrm{~Hz} ;\right.$ ipso-Ph $), 142.5\left(\mathrm{~d},{ }^{1} J(\mathrm{C}, \mathrm{P})=\right.$ $31.2 \mathrm{~Hz} ; \mathrm{PC}=\mathrm{C}), 172.0\left(\mathrm{~d},{ }^{2} J(\mathrm{C}, \mathrm{P})=5.8 \mathrm{~Hz} ; \mathrm{PC}=C\right), 196.8\left(\mathrm{~d},{ }^{2} J(\mathrm{C}, \mathrm{P})=\right.$ $7.1,{ }^{1} J(\mathrm{C}, \mathrm{W})=125.4 \mathrm{~Hz}$; cis-CO $), 199.3 \mathrm{ppm}\left(\mathrm{d},{ }^{2} J(\mathrm{C}, \mathrm{P})=21.1 \mathrm{~Hz}\right.$; trans$\mathrm{CO}) ;{ }^{31} \mathrm{P}\left\{{ }^{1} \mathrm{H}\right\} \quad \mathrm{NMR} \quad\left(101.3 \mathrm{MHz}, \quad \mathrm{CDCl}_{3}\right): \quad \delta=34.4 \mathrm{ppm} \quad\left({ }^{1} \mathrm{~J}(\mathrm{P}, \mathrm{W})=\right.$ $238.4 \mathrm{~Hz}$ ); IR (KBr): $\tilde{v}=1908,1933\left(\mathrm{~s} / \mathrm{br}, \mathrm{CO}_{\mathrm{eq}}\right), 1977 \quad\left(\mathrm{w}, \mathrm{CO}_{\mathrm{eq}}\right)$, $2068 \mathrm{~cm}^{-1}\left(\mathrm{~m}, \mathrm{CO}_{\mathrm{ax}}\right)$; MS (70 eV): $m / z(\%): 616(2)[M]^{+}$; HR-MS (EI, $70 \mathrm{eV}$ ): $\mathrm{m} / z$ : calcd for $\mathrm{C}_{25} \mathrm{H}_{21} \mathrm{O}_{5} \mathrm{P}^{184} \mathrm{~W}$ : 616.06366 ; found: 616.06445

\section{(8-Phenyl-8-phosphatrispiro[2.0.0.2.1.1]non-8-yl)pentacarbonyltungsten} (21): Complex 8 (369 mg, $0.55 \mathrm{mmol}$ ), cyclopropylidenespiropentane (20) $(117 \mathrm{mg}, 1.10 \mathrm{mmol})$ and $\mathrm{CuCl}(10 \mathrm{mg}, 0.1 \mathrm{mmol})$ were heated in toluene $(4 \mathrm{~mL})$ at $55^{\circ} \mathrm{C}$ for $1 \mathrm{~h}$. Evaporation to dryness and chromatography of the residue over silica gel eluting with pentane/dichloromethane 19:1 gave $21 \mathbf{a}$ and $\mathbf{b}$ in a $4: 3$ ratio $(220 \mathrm{mg}, 78 \%)$ as a pale yellow oil. Fractional recrystallization from pentane at $-20^{\circ} \mathrm{C}$ afforded colorless crystals of both isomers.

anti-(4R,9R)- and $\quad(\mathbf{4 S , 9 S )})$-Isomer $\quad 21 \mathrm{a}: \quad$ m.p. $\quad 99^{\circ} \mathrm{C} ; \quad{ }^{1} \mathrm{H} N M R$ $\left(400.13 \mathrm{MHz}, \mathrm{CDCl}_{3}\right): \delta=0.43-0.48(\mathrm{~m}, 1 \mathrm{H} ; \mathrm{CH}), 0.76-0.91(\mathrm{~m}, 3 \mathrm{H}$; $\mathrm{CH}), 0.93-1.00(\mathrm{~m}, 1 \mathrm{H} ; \mathrm{PCCH}), 1.13\left(\mathrm{dd},{ }^{2} \mathrm{~J}(\mathrm{H}, \mathrm{H})=4.3,{ }^{2} J(\mathrm{H}, \mathrm{P})=7.0 \mathrm{~Hz}\right.$, $1 \mathrm{H}, \mathrm{PCC} H \mathrm{C}), 1.35-1.46(\mathrm{~m}, 2 \mathrm{H} ; \mathrm{PCC} H), 1.47-1.53(\mathrm{~m}, 1 \mathrm{H} ; \mathrm{PCC} H)$, $1.67\left(\mathrm{dd},{ }^{2} J(\mathrm{H}, \mathrm{H})=4.3,{ }^{2} J(\mathrm{H}, \mathrm{P})=10.9 \mathrm{~Hz}, 1 \mathrm{H} ; \mathrm{PCCHC}\right), 7.35-7.46 \mathrm{ppm}$ $\left.(\mathrm{m}, 5 \mathrm{H} ; \mathrm{Ph} H) ;{ }^{13} \mathrm{C}^{1} \mathrm{H}\right\}$ NMR $\left(100.62 \mathrm{MHz}, \mathrm{CDCl}_{3}\right): \delta=3.9\left(\mathrm{~d},{ }^{3} J(\mathrm{C}, \mathrm{P})=\right.$ $\left.4.6 \mathrm{~Hz} ; \mathrm{CH}_{2}\right), 4.7\left(\mathrm{~s} ; \mathrm{CH}_{2}\right), 7.4\left(\mathrm{~d},{ }^{2} \mathrm{~J}(\mathrm{C}, \mathrm{P})=3.6 \mathrm{~Hz} ; \mathrm{PCCH}_{2}\right), 8.9(\mathrm{~s} ;$ $\left.\mathrm{PCCH}_{2}\right), 15.0$ (s; $\left.\mathrm{PCCH}_{2} \mathrm{C}\right), 18.0\left(\mathrm{~d},{ }^{2} J(\mathrm{C}, \mathrm{P})=5.3 \mathrm{~Hz} ; \mathrm{PCC}\right), 24.8(\mathrm{~s} ; \mathrm{PC})$, $30.1\left(\mathrm{~d},{ }^{1} J(\mathrm{C}, \mathrm{P})=2.3 \mathrm{~Hz} ; \mathrm{PC}\right), 128.4\left(\mathrm{~d},{ }^{3} J(\mathrm{C}, \mathrm{P})=10.5 \mathrm{~Hz} ; m-\mathrm{Ph}\right), 130.2$ $\left(\mathrm{d},{ }^{4} J(\mathrm{C}, \mathrm{P})=2.6 \mathrm{~Hz} ; p-\mathrm{Ph}\right), 132.2\left(\mathrm{~d},{ }^{2} J(\mathrm{C}, \mathrm{P})=13.5 \mathrm{~Hz} ; o-\mathrm{Ph}\right), 132.6\left(\mathrm{~d},{ }^{1} J-\right.$ $(\mathrm{C}, \mathrm{P})=21.5 \mathrm{~Hz} ;$ ipso-Ph), $195.6\left(\mathrm{~d},{ }^{2} J(\mathrm{C}, \mathrm{P})=8.4,{ }^{1} J(\mathrm{C}, \mathrm{W})=125.4 \mathrm{~Hz} ;\right.$ cisCO), $197.8 \mathrm{ppm}\left(\mathrm{d},{ }^{2} J(\mathrm{C}, \mathrm{P})=29.4 \mathrm{~Hz}\right.$; trans-CO); ${ }^{31} \mathrm{P}$ NMR $(101.25 \mathrm{MHz}$, $\left.\mathrm{CDCl}_{3}\right): \delta=-123.9 \mathrm{ppm}\left({ }^{1} J(\mathrm{P}, \mathrm{W})=257.2 \mathrm{~Hz}\right)$; IR $(\mathrm{KBr}): \tilde{v}=1914$ and 1931 (s/br, $\left.\mathrm{CO}_{\mathrm{eq}}\right), 1987$ (w, $\left.\mathrm{CO}_{\mathrm{eq}}\right), 2072 \mathrm{~cm}^{-1}\left(\mathrm{~m}, \mathrm{CO}_{\mathrm{ax}}\right) ; \mathrm{MS}(70 \mathrm{eV}): \mathrm{m} / \mathrm{z}$ (\%): $538(2)[M]^{+}, 454(3)[M-3 \mathrm{CO}]^{+}, 426(6)[M-4 \mathrm{CO}]^{+}, 398(20)$ $[M-5 \mathrm{CO}]^{+}$; HR-MS (EI): $m / z$ : calcd for $\mathrm{C}_{19} \mathrm{H}_{15} \mathrm{O}_{5} \mathrm{P}^{184} \mathrm{~W}: 538.01672$; found: 538.01570; elemental analysis calcd (\%) for $\mathrm{C}_{19} \mathrm{H}_{15} \mathrm{O}_{5} \mathrm{P}^{184} \mathrm{~W}$ : $\mathrm{C}$ 42.41, H 2.81; found: C 42.24, H 2.82.

syn-(4S,9R)- and (4R,9S)-isomer $21 \mathrm{~b}$ : m.p. $\quad 74-75^{\circ} \mathrm{C} ; \quad{ }^{1} \mathrm{H} N M R$ $\left(400.13 \mathrm{MHz}, \mathrm{CDCl}_{3}\right): \delta=0.77-0.84(\mathrm{~m}, 1 \mathrm{H} ; \mathrm{CH}), 0.95-1.07(\mathrm{~m}, 2 \mathrm{H}$; $\mathrm{CH}), 1.10-1.20(\mathrm{~m}, 2 \mathrm{H} ; \mathrm{CH}, \mathrm{PCC} H), 1.22-1.30(\mathrm{~m}, 2 \mathrm{H} ; \mathrm{PCC}), 1.32$ $\left(\mathrm{dd},{ }^{2} J(\mathrm{H}, \mathrm{H})=4.2,{ }^{2} J(\mathrm{H}, \mathrm{P})=2.6 \mathrm{~Hz}, 1 \mathrm{H} ; \mathrm{PCC} H \mathrm{C}\right), 1.34-1.44(\mathrm{~m}, 1 \mathrm{H}$; $\mathrm{PCCH}), 1.51\left(\mathrm{dd},{ }^{2} J(\mathrm{H}, \mathrm{H})=4.2,{ }^{2} J(\mathrm{H}, \mathrm{P})=11.9 \mathrm{~Hz}, 1 \mathrm{H} ; \mathrm{PCC} H \mathrm{C}\right), 7.38-$ $7.46(\mathrm{~m}, 3 \mathrm{H} ; m-\mathrm{Ph} H, p-\mathrm{Ph} H), 7.56-7.64 \mathrm{ppm}(\mathrm{m}, 2 \mathrm{H} ; o-\mathrm{Ph} H) ;{ }^{13} \mathrm{C}\left\{{ }^{1} \mathrm{H}\right\}$ NMR $\left(100.62 \mathrm{MHz}, \mathrm{CDCl}_{3}\right): \delta=4.1\left(\mathrm{~d},{ }^{3} \mathrm{~J}(\mathrm{C}, \mathrm{P})=2.4 \mathrm{~Hz} ; \mathrm{CH}_{2}\right), 5.2\left(\mathrm{~d},{ }^{3} \mathrm{~J}-\right.$ $\left.(\mathrm{C}, \mathrm{P})=3.7 \mathrm{~Hz} ; \mathrm{CH}_{2}\right), 8.0\left(\mathrm{~d},{ }^{2} J(\mathrm{C}, \mathrm{P})=3.5 \mathrm{~Hz} ; \mathrm{PCCH}_{2}\right), 8.2\left(\mathrm{~s} ; \mathrm{PCCH}_{2}\right)$, $14.9\left(\mathrm{~d},{ }^{2} \mathrm{~J}(\mathrm{C}, \mathrm{P})=3.8 \mathrm{~Hz} ; \mathrm{PCCH}_{2} \mathrm{C}\right), 18.8(\mathrm{~s} ; \mathrm{PCC}), 25.3$ (s; PC), 30.0 (d, $\left.{ }^{1} J(\mathrm{C}, \mathrm{P})=3.5 \mathrm{~Hz} ; \mathrm{PC}\right), 128.5\left(\mathrm{~d},{ }^{3} J(\mathrm{C}, \mathrm{P})=10.5 \mathrm{~Hz} ; m-\mathrm{Ph}\right), 130.2\left(\mathrm{~d},{ }^{4} J-\right.$ $(\mathrm{C}, \mathrm{P})=2.6 \mathrm{~Hz} ; p-\mathrm{Ph}), 132.3\left(\mathrm{~d},{ }^{2} J(\mathrm{C}, \mathrm{P})=13.4 \mathrm{~Hz} ; o-\mathrm{Ph}\right), 134.0\left(\mathrm{~d},{ }^{1} J-\right.$ $(\mathrm{C}, \mathrm{P})=20.7 \mathrm{~Hz} ;$ ipso-Ph), $195.7\left(\mathrm{~d},{ }^{2} J(\mathrm{C}, \mathrm{P})=8.3,{ }^{1} J(\mathrm{C}, \mathrm{W})=125.4 \mathrm{~Hz} ;\right.$ cisCO), $197.7 \mathrm{ppm}\left(\mathrm{d},{ }^{2} J(\mathrm{C}, \mathrm{P})=29.4 \mathrm{~Hz}\right.$; trans-CO $) ;{ }^{31} \mathrm{P}$ NMR $(101.25 \mathrm{MHz}$, $\left.\mathrm{CDCl}_{3}\right): \delta=-128.1 \mathrm{ppm}\left({ }^{1} J(\mathrm{P}, \mathrm{W})=254.1 \mathrm{~Hz}\right) ; \mathrm{IR}(\mathrm{KBr}): \tilde{v}=1937(\mathrm{~s} / \mathrm{br}$, $\mathrm{CO}_{\mathrm{eq}}$ ), 1987 (w, $\mathrm{CO}_{\mathrm{eq}}$ ), $2072 \mathrm{~cm}^{-1}\left(\mathrm{w}, \mathrm{CO}_{\mathrm{ax}}\right.$ ).

Synthesis of $\mathbf{2 3}$ and 24: Complex $\mathbf{8}(0.33 \mathrm{~g}, \quad 0.50 \mathrm{mmol})$, 7 cyclopropylidenedispiro[2.0.2.1]heptane $(\mathbf{2 2}) \quad(0.13 \mathrm{~g}, 1.00 \mathrm{mmol})$ and $\mathrm{CuCl}(10 \mathrm{mg}, 0.1 \mathrm{mmol})$ were stirred at room temperature for $20 \mathrm{~h}$ in toluene $(4 \mathrm{~mL})$. Evaporation to dryness and chromatography of the residue over silica gel eluting with pentane/dichloromethane $9: 1$ yielded $\mathbf{2 3}$ and 24 as a pale yellow solid. Fractional recrystallization from pentane at $0{ }^{\circ} \mathrm{C}$ afforded colorless crystals of $\mathbf{2 3}(200 \mathrm{mg}, 71 \%)$. $\mathbf{2 4}$ was obtained as a col- orless oil (5 mg, $2 \%$ ) after sequential column chromatography over silica gel eluting with pentane/dichloromethane 9:1.

(11-Phenyl-11-phosphatetraspiro[2.0.2.0.0.2.1] undec-11-yl)pentacarbonyltungsten (23): m.p. $93^{\circ} \mathrm{C}$; ${ }^{1} \mathrm{H}$ NMR $\left(400.13 \mathrm{MHz}, \mathrm{CDCl}_{3}\right): \delta=0.19-0.24$ $(\mathrm{m}, 1 \mathrm{H} ; \mathrm{CH}), 0.49-0.57(\mathrm{~m}, 1 \mathrm{H} ; \mathrm{CH}), 0.58-0.64(\mathrm{~m}, 2 \mathrm{H} ; \mathrm{CH}), 0.76-0.86$ $(\mathrm{m}, 2 \mathrm{H} ; \mathrm{CH}), 0.96-1.02(\mathrm{~m}, 1 \mathrm{H} ; \mathrm{CH}), 1.10-1.27\left(\mathrm{~m}, 3 \mathrm{H} ; \mathrm{CH}, \mathrm{PCCH}_{2}\right)$, 1.47-1.61 (m, 2H; $\left.\mathrm{PCCH}_{2}\right), 7.35-7.40(\mathrm{~m}, 3 \mathrm{H} ; m-\mathrm{Ph} H, p-\mathrm{Ph} H), 7.48-$ $7.54 \mathrm{ppm}(\mathrm{m}, 2 \mathrm{H} ; o-\mathrm{Ph} H) ;{ }^{13} \mathrm{C}\left\{{ }^{1} \mathrm{H}\right\} \mathrm{NMR}\left(100.62 \mathrm{MHz}, \mathrm{CDCl}_{3}\right): \delta=3.0$ $\left(\mathrm{d},{ }^{3} \mathrm{~J}(\mathrm{C}, \mathrm{P})=4.0 \mathrm{~Hz} ; \mathrm{CH}_{2}\right), 3.5\left(\mathrm{~d},{ }^{3} \mathrm{~J}(\mathrm{C}, \mathrm{P})=1.6 \mathrm{~Hz} ; \mathrm{CH}_{2}\right), 3.9\left(\mathrm{~s} ; \mathrm{CH}_{2}\right), 4.6$ $\left(\mathrm{d},{ }^{3} J(\mathrm{C}, \mathrm{P})=3.2 \mathrm{~Hz} ; \mathrm{CH}_{2}\right), 6.8\left(\mathrm{~s} ; \mathrm{PCCH}_{2}\right), 6.9\left(\mathrm{~d},{ }^{2} J(\mathrm{C}, \mathrm{P})=3.3 \mathrm{~Hz}\right.$; $\left.\mathrm{PCCH}_{2}\right), 20.8\left(\mathrm{~d},{ }^{2} J(\mathrm{C}, \mathrm{P})=1.7 \mathrm{~Hz} ; \mathrm{PCC}\right), 21.5\left(\mathrm{~d},{ }^{2} J(\mathrm{C}, \mathrm{P})=5.6 \mathrm{~Hz} ; \mathrm{PCC}\right)$, $23.5\left(\mathrm{~s} ; \mathrm{PC}\left(\mathrm{CH}_{2}\right)_{2}\right), 32.2\left(\mathrm{~d},{ }^{1} J(\mathrm{C}, \mathrm{P})=5.7 \mathrm{~Hz} ; \mathrm{PC}\right), 128.4\left(\mathrm{~d},{ }^{3} J(\mathrm{C}, \mathrm{P})=\right.$ $10.5 \mathrm{~Hz} ; m-\mathrm{Ph}), 130.2\left(\mathrm{~d},{ }^{4} J(\mathrm{C}, \mathrm{P})=2.6 \mathrm{~Hz} ; p-\mathrm{Ph}\right), 132.0\left(\mathrm{~d},{ }^{2} J(\mathrm{C}, \mathrm{P})=\right.$ $13.2 \mathrm{~Hz} ; o-\mathrm{Ph}), 133.1\left(\mathrm{~d},{ }^{1} J(\mathrm{C}, \mathrm{P})=21.0 \mathrm{~Hz}\right.$; ipso-Ph), $195.7\left(\mathrm{~d},{ }^{2} J(\mathrm{C}, \mathrm{P})=\right.$ $8.4,{ }^{1} J(\mathrm{C}, \mathrm{W})=125.5 \mathrm{~Hz} ;$ cis-CO $), 197.9 \mathrm{ppm}\left(\mathrm{d},{ }^{2} J(\mathrm{C}, \mathrm{P})=29.4,{ }^{1} J(\mathrm{C}, \mathrm{W})=\right.$ $149.6 \mathrm{~Hz}$; trans-CO); ${ }^{31} \mathrm{P}$ NMR $\left(101.25 \mathrm{MHz}, \mathrm{CDCl}_{3}\right): \delta=-124.1 \mathrm{ppm}\left({ }^{1} J-\right.$ $(\mathrm{P}, \mathrm{W})=250.6 \mathrm{~Hz})$; IR $(\mathrm{KBr}): \tilde{v}=1915,1933\left(\mathrm{~s} / \mathrm{br}, \mathrm{CO}_{\mathrm{eq}}\right), 1979\left(\mathrm{~m}, \mathrm{CO}_{\mathrm{eq}}\right)$, $2072 \mathrm{~cm}^{-1}\left(\mathrm{~m}, \mathrm{CO}_{\mathrm{ax}}\right)$; MS (70 eV): $\mathrm{m} / z$ (\%): $564(13)[M]^{+}, 508(2)$ $[M-2 \mathrm{CO}]^{+}, 480$ (7) $[M-3 \mathrm{CO}]^{+}, 452$ (19) $[M-4 \mathrm{CO}]^{+}, 424$ (100) [M-5CO $]^{+}$; HR-MS (EI): $m / z$ : calcd for $\mathrm{C}_{21} \mathrm{H}_{17} \mathrm{O}_{5} \mathrm{P}^{184} \mathrm{~W}: 564.03235$; found: 564.03512 .

\{2-Phenyl-2-phospha-3:3,4:4-bisethanobicyclo[3.2.0]hept-1(5)-en-2-yl\}pentacarbonyltungsten (24): ${ }^{1} \mathrm{H}$ NMR $\left(400.1 \mathrm{MHz}, \mathrm{CDCl}_{3}\right): \delta=0.21-0.31$ (m, 2H; PCCCH, PCCH), 0.32-0.40 (m, 1 H; PCCH), 0.49-0.55 (m, 1 H; $\mathrm{PCC} H), 0.62-0.74(\mathrm{~m}, 3 \mathrm{H} ; \mathrm{PCCCH}), 0.90-0.99(\mathrm{~m}, 1 \mathrm{H}, \mathrm{PCCH}), 2.79-$ $2.91(\mathrm{~m}, 2 \mathrm{H} ;=\mathrm{CCH}), 2.96-3.12(\mathrm{~m}, 2 \mathrm{H},=\mathrm{CCH}), 7.40-7.51 \mathrm{ppm}(\mathrm{m}, 5 \mathrm{H}$; $\mathrm{Ph} H) ;{ }^{13} \mathrm{C}\left\{{ }^{1} \mathrm{H}\right\}$ NMR $\left(100.6 \mathrm{MHz}, \mathrm{CDCl}_{3}\right): \delta=7.7,8.9\left(\mathrm{~s} ; \mathrm{PCCCH}_{2}\right), 10.8$ $\left(\mathrm{d},{ }^{2} J(\mathrm{C}, \mathrm{P})=3.1 \mathrm{~Hz} ; \mathrm{PCCH}_{2}\right), 11.1\left(\mathrm{~d},{ }^{2} J(\mathrm{C}, \mathrm{P})=7.4 \mathrm{~Hz} ; \mathrm{PCCH}_{2}\right), 28.5(\mathrm{~d}$, $\left.{ }^{2} J(\mathrm{C}, \mathrm{P})=2.5 \mathrm{~Hz} ; \mathrm{P}(\mathrm{C}=) \mathrm{CH}_{2}\right), 28.7\left(\mathrm{~d},{ }^{3} J(\mathrm{C}, \mathrm{P})=15.8 \mathrm{~Hz} ; \mathrm{PC}=\mathrm{CCH}_{2}\right), 31.4$ $\left(\mathrm{d},{ }^{2} J(\mathrm{C}, \mathrm{P})=8.2 \mathrm{~Hz} ; \mathrm{PCC}\right), 33.1\left(\mathrm{~d},{ }^{1} J(\mathrm{C}, \mathrm{P})=40.2 \mathrm{~Hz} ; \mathrm{PC}\right), 128.6\left(\mathrm{~d},{ }^{3} J-\right.$ $(\mathrm{C}, \mathrm{P})=9.6 \mathrm{~Hz} ; m-\mathrm{Ph}), 130.4\left(\mathrm{~d},{ }^{4} J(\mathrm{C}, \mathrm{P})=2.1 \mathrm{~Hz} ; p-\mathrm{Ph}\right), 131.6\left(\mathrm{~d},{ }^{2} J-\right.$ $(\mathrm{C}, \mathrm{P})=12.2 \mathrm{~Hz} ; o-\mathrm{Ph}), 136.0\left(\mathrm{~d},{ }^{1} J(\mathrm{C}, \mathrm{P})=32.3 \mathrm{~Hz} ;\right.$ ipso $\left.-\mathrm{Ph}\right), 140.3\left(\mathrm{~d},{ }^{1} J-\right.$ $(\mathrm{C}, \mathrm{P})=29.7 \mathrm{~Hz} ; \mathrm{PC}=\mathrm{C}), 170.6\left(\mathrm{~d},{ }^{2} J(\mathrm{C}, \mathrm{P})=4.4 \mathrm{~Hz} ; \mathrm{PC}=C\right), 196.7\left(\mathrm{~d},{ }^{2} J-\right.$ $(\mathrm{C}, \mathrm{P})=7.1,{ }^{1} J(\mathrm{C}, \mathrm{W})=125.4 \mathrm{~Hz} ;$ cis $\left.-\mathrm{CO}\right), 199.5 \mathrm{ppm}\left(\mathrm{d},{ }^{2} J(\mathrm{C}, \mathrm{P})=21.0 \mathrm{~Hz}\right.$; trans-CO); ${ }^{31} \mathrm{P}$ NMR $\quad\left(101.25 \mathrm{MHz}, \quad \mathrm{CDCl}_{3}\right): \quad \delta=34.6 \mathrm{ppm} \quad\left({ }^{1} J_{\mathrm{P}, \mathrm{W}}=\right.$ $234.7 \mathrm{~Hz}) ; \mathrm{MS}(70 \mathrm{eV}): m / z(\%): 564(10)[M]^{+}, 508(2)[M-2 \mathrm{CO}]^{+}, 480$ (5) $[M-3 \mathrm{CO}]^{+}, 452(4)[M-4 \mathrm{CO}]^{+}, 424(20)[M-5 \mathrm{CO}]^{+}$; HR-MS (EI): calcd for $\mathrm{C}_{21} \mathrm{H}_{17} \mathrm{O}_{5} \mathrm{P}^{184} \mathrm{~W}$ : 564.03235 ; found: 564.03554 .

Synthesis of 26 and 27: Complex $8(600 \mathrm{mg}, 0.92 \mathrm{mmol})$, ethenylbicyclopropylidene $(\mathbf{2 5})(293 \mathrm{mg}, 2.76 \mathrm{mmol})$ and $\mathrm{CuCl}(10 \mathrm{mg}, 0.1 \mathrm{mmol})$ were stirred in toluene $(4 \mathrm{~mL})$ at room temperature for $18 \mathrm{~h}$. Evaporation to dryness and chromatography of the residue over silica gel eluting with pentane/dichloromethane 19:1 gave a mixture of six phosphirane isomers (440 mg, $89 \%$ ) as a pale yellow oil. This mixture consisted of $9 \%$ of the phospha[3]triangulane isomers $26 \mathbf{a}$ and b (1:1 ratio) and $91 \%$ of the phosphiranes 27 (four isomers 6:11:17:66). Colorless crystals of $\mathbf{2 7} \mathbf{d}$ could be isolated by fractional recrystallization from hexane at $-80^{\circ} \mathrm{C}$ followed by recrystallization at $-80^{\circ} \mathrm{C}$. $\mathbf{2 6}$ a could be separated from the remaining isomers by subsequent column chromatography over silica gel eluting with pentane/dichloromethane $19: 1$ and recrystallization at $-80^{\circ} \mathrm{C}$.

(1-Ethenyl-7-phenyl-7-phosphadispiro[2.0.2.1]hept-7-yl)pentacarbonyltungsten (26)

anti-(1R,3S,7S)- and (1S,3R,7R)-Isomer $26 \mathrm{a}:{ }^{1} \mathrm{H}$ NMR $(400.13 \mathrm{MHz}$, $\left.\mathrm{CDCl}_{3}\right): \delta=0.90-1.02(\mathrm{~m}, 1 \mathrm{H} ; \mathrm{CH}), 1.02-1.08\left(\mathrm{~m},{ }^{2} J(\mathrm{H}, \mathrm{H})=5.3 \mathrm{~Hz}, 1 \mathrm{H}\right.$; $\mathrm{CH}), 1.15-1.25(\mathrm{~m}, 1 \mathrm{H} ; \mathrm{CH}), 1.40-1.50(\mathrm{~m}, 1 \mathrm{H} ; \mathrm{CH}), 1.50-1.61(\mathrm{~m}, 1 \mathrm{H}$; $\mathrm{CH}), 1.74-1.81\left(\mathrm{~m},{ }^{2} \mathrm{~J}(\mathrm{H}, \mathrm{H})=5.3 \mathrm{~Hz}, 1 \mathrm{H} ; \mathrm{CH}\right), 2.37-2.43(\mathrm{~m}, 1 \mathrm{H}$; $\mathrm{PCCH}), 5.08-5.11\left(\mathrm{~m}, 1 \mathrm{H} ;=\mathrm{CH}_{2}\right), 5.24-5.28\left(\mathrm{~m}, 2 \mathrm{H} ;=\mathrm{CH}_{2},=\mathrm{CH}\right), 7.42-$ $7.46(\mathrm{~m}, 3 \mathrm{H} ; m-\mathrm{Ph} H, p-\mathrm{Ph} H), 7.56-7.62 \mathrm{ppm}(\mathrm{m}, 2 \mathrm{H} ; o-\mathrm{Ph} H) ;{ }^{13} \mathrm{C}\left\{{ }^{1} \mathrm{H}\right\}$ NMR (100.62 MHz, $\left.\mathrm{CDCl}_{3}\right): \delta=7.6\left(\mathrm{~d},{ }^{2} J(\mathrm{C}, \mathrm{P})=3.4 \mathrm{~Hz} ; \mathrm{CH}_{2}\right), 9.8(\mathrm{~s}$; $\left.\mathrm{CH}_{2}\right), 17.3\left(\mathrm{~s} ; \mathrm{CH}_{2}\right), 26.5(\mathrm{~s} ; \mathrm{PC}), 27.1\left(\mathrm{~d},{ }^{2} \mathrm{~J}(\mathrm{C}, \mathrm{P})=3.1 \mathrm{~Hz} ; \mathrm{PCCH}\right), 31.0$ $\left(\mathrm{d},{ }^{1} J(\mathrm{C}, \mathrm{P})=2.2 \mathrm{~Hz} ; \mathrm{PC}\right), 115.9\left(\mathrm{~s} ;=\mathrm{CH}_{2}\right), 128.5\left(\mathrm{~d},{ }^{3} J(\mathrm{C}, \mathrm{P})=10.9 \mathrm{~Hz} ; m-\right.$ $\mathrm{Ph}), 130.4\left(\mathrm{~d},{ }^{4} J(\mathrm{C}, \mathrm{P})=2.7 \mathrm{~Hz} ; p-\mathrm{Ph}\right), 132.7$ (d, ${ }^{1} J(\mathrm{C}, \mathrm{P})=21.6 \mathrm{~Hz}$; ipso$\mathrm{Ph}), 133.1\left(\mathrm{~d},{ }^{2} J(\mathrm{C}, \mathrm{P})=14.2 \mathrm{~Hz} ; o-\mathrm{Ph}\right), 137.6(\mathrm{~s} ;=\mathrm{CH}), 195.5\left(\mathrm{~d},{ }^{2} J-\right.$ $(\mathrm{C}, \mathrm{P})=8.3 \mathrm{~Hz} ;$ cis-CO), $197.5 \mathrm{ppm} \quad\left(\mathrm{d},{ }^{2} J(\mathrm{C}, \mathrm{P})=29.5 \mathrm{~Hz} ;\right.$ trans-CO $)$; ${ }^{31} \mathrm{P}$ NMR $\left(101.25 \mathrm{MHz}, \mathrm{CDCl}_{3}\right): \delta=-127.0 \mathrm{ppm}\left({ }^{1} J(\mathrm{P}, \mathrm{W})=259.4 \mathrm{~Hz}\right)$.

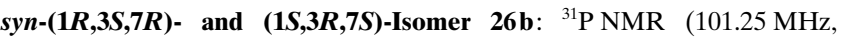
$\left.\mathrm{CDCl}_{3}\right): \delta=-129.8 \mathrm{ppm}\left({ }^{1} J(\mathrm{P}, \mathrm{W})=260.4 \mathrm{~Hz}\right)$. 
(1-Phenyl-2-bicyclopropylidenylphosphiranyl)pentacarbonyltungsten (27) Compound 27a: ${ }^{31} \mathrm{P}$ NMR $\left(101.25 \mathrm{MHz}, \mathrm{CDCl}_{3}\right): \delta=-165.7 \mathrm{ppm} \quad\left({ }^{1} J-\right.$ $(\mathrm{P}, \mathrm{W})=257.8 \mathrm{~Hz})$.

Compound 27b: ${ }^{31} \mathrm{P}$ NMR $\left(101.25 \mathrm{MHz}, \mathrm{CDCl}_{3}\right): \delta=-167.7 \mathrm{ppm}\left({ }^{1} J-\right.$ $(\mathrm{P}, \mathrm{W})=258.0 \mathrm{~Hz})$.

Compound 27c: ${ }^{31} \mathrm{P}$ NMR $\left(101.25 \mathrm{MHz}, \mathrm{CDCl}_{3}\right): \delta=-167.7 \mathrm{ppm} \quad\left({ }^{1} J-\right.$ $(\mathrm{P}, \mathrm{W})=252.8 \mathrm{~Hz})$.

anti-(1S,2S,4R)- and $(1 R, 2 R, 4 S)$-Isomer $27 \mathrm{~d}$ : m.p. $\quad 61{ }^{\circ} \mathrm{C} ; \quad{ }^{1} \mathrm{H}$ NMR $\left(400.13 \mathrm{MHz}, \mathrm{CDCl}_{3}\right): \delta=0.73-0.81(\mathrm{~m}, 2 \mathrm{H}$; $=\mathrm{CCH}), 1.01-1.13(\mathrm{~m}, 2 \mathrm{H}$; $=\mathrm{CC} H), 1.13-1.20\left(\mathrm{~m}, 1 \mathrm{H} ; \mathrm{PCCCH}_{2}\right), 1.32-1.37(\mathrm{~m}, 1 \mathrm{H} ; \mathrm{PCC} H), 1.40$ $1.46\left(\mathrm{~m}, 1 \mathrm{H} ; \mathrm{PCCCH}_{2}\right), 1.52-1.58\left(\mathrm{~m}, 1 \mathrm{H} ; \mathrm{PCH}_{2}\right), 1.64-1.70(\mathrm{~m}, 1 \mathrm{H}$; $\mathrm{PCH}), 1.70-1.76\left(\mathrm{~m}, 1 \mathrm{H} ; \mathrm{PCH}_{2}\right), 7.35-7.42(\mathrm{~m}, 3 \mathrm{H} ; m-\mathrm{Ph} H, p-\mathrm{Ph} H)$, 7.50-7.62 ppm (m, 2H;o-PhH); ${ }^{13} \mathrm{C}\left\{{ }^{1} \mathrm{H}\right\}$ NMR (100.62 MHz, $\left.\mathrm{CDCl}_{3}\right): \delta=$ 2.8, $3.0\left(2 \mathrm{~s} ;=\mathrm{C}\left(\mathrm{CH}_{2}\right)_{2}\right), 10.4\left(\mathrm{~d},{ }^{3} J(\mathrm{C}, \mathrm{P})=5.0 \mathrm{~Hz} ; \mathrm{PCCCH}_{2}\right), 14.7\left(\mathrm{~d},{ }^{1} J-\right.$ $\left.(\mathrm{C}, \mathrm{P})=11.8 \mathrm{~Hz} ; \mathrm{PCH}_{2}\right), 16.3\left(\mathrm{~d},{ }^{2} J(\mathrm{C}, \mathrm{P})=4.0 \mathrm{~Hz} ; \mathrm{PCCH}\right), 28.5\left(\mathrm{~d},{ }^{1} J-\right.$ $(\mathrm{C}, \mathrm{P})=16.3 \mathrm{~Hz} ; \mathrm{PCH}), 112.9\left(\mathrm{~s} ;=C\left(\mathrm{CH}_{2}\right)_{2}\right), 113.3\left(\mathrm{~d},{ }^{3} J(\mathrm{C}, \mathrm{P})=6.2 \mathrm{~Hz}\right.$; $\mathrm{PCCC}=), 128.8\left(\mathrm{~d},{ }^{3} J(\mathrm{C}, \mathrm{P})=10.0 \mathrm{~Hz} ; m-\mathrm{Ph}\right), 130.2\left(\mathrm{~d},{ }^{4} J(\mathrm{C}, \mathrm{P})=2.1 \mathrm{~Hz} ; p\right.$ $\mathrm{Ph}), 132.6\left(\mathrm{~d},{ }^{1} J(\mathrm{C}, \mathrm{P})=30.8 \mathrm{~Hz}\right.$; ipso-Ph $), 132.8\left(\mathrm{~d},{ }^{2} J(\mathrm{C}, \mathrm{P})=11.6 \mathrm{~Hz} ; o-\right.$ $\mathrm{Ph}), 195.8\left(\mathrm{~d},{ }^{2} J(\mathrm{C}, \mathrm{P})=8.3,{ }^{1} J(\mathrm{C}, \mathrm{W})=125.7 \mathrm{~Hz}\right.$; cis-CO), $198.4 \mathrm{ppm}\left(\mathrm{d},{ }^{2} J-\right.$ $(\mathrm{C}, \mathrm{P})=29.9 \mathrm{~Hz} ; \quad$ trans $-\mathrm{CO}) ; \quad{ }^{31} \mathrm{P} \mathrm{NMR} \quad\left(101.25 \mathrm{MHz}, \quad \mathrm{CDCl}_{3}\right): \quad \delta=$ $-169.5 \mathrm{ppm}\left({ }^{1} J(\mathrm{P}, \mathrm{W})=253.0 \mathrm{~Hz}\right)$; IR $(\mathrm{KBr}): \tilde{v}=1923\left(\mathrm{~s} / \mathrm{br}, \mathrm{CO}_{\mathrm{eq}}\right), 1985$ $\left(\mathrm{w}, \mathrm{CO}_{\mathrm{eq}}\right), 2072 \mathrm{~cm}^{-1}\left(\mathrm{w}, \mathrm{CO}_{\mathrm{ax}}\right) ; \mathrm{MS}(70 \mathrm{eV}): \mathrm{m} / z(\%): 538(3)[M]^{+}, 482$ (6) $[M-2 \mathrm{CO}]^{+}, 454(8)[M-3 \mathrm{CO}]^{+}, 426(23)[M-4 \mathrm{CO}]^{+}, 398(85)$ $[M-5 \mathrm{CO}]^{+}$; HR-MS (EI): $m / z$ : calcd for $\mathrm{C}_{19} \mathrm{H}_{15} \mathrm{O}_{5} \mathrm{P}^{184} \mathrm{~W}: 538.01672$; found: 538.01634 .

\{1-(1'-Phenyl-1'-pentacarbonyltungstenphosphiran-2-yl)-7-phenyl-7-phosphadispiro[2.0.2.1] hept-7-yl\}pentacarbonyltungsten (28): Compound 27d $(55 \mathrm{mg}, 0.10 \mathrm{mmol})$, complex $8(133 \mathrm{mg}, 0.20 \mathrm{mmol})$ and $\mathrm{CuCl}(10 \mathrm{mg}$, $0.1 \mathrm{mmol})$ were heated in toluene $(1 \mathrm{~mL})$ at $55^{\circ} \mathrm{C}$ for $6.5 \mathrm{~h}$. Evaporation to dryness and chromatography of the residue over silica gel eluting with pentane/dichloromethane $4: 1$ yielded $\mathbf{2 8} \mathbf{a}$ and b in a $5: 3$ ratio $(60 \mathrm{mg}$, $62 \%)$ as a pale yellow oil; $12 \%$ of $\mathbf{2 7} \mathbf{d}(7 \mathrm{mg})$ could be recovered. Subsequent chromatography on silica gel eluting with pentane/dichloromethane 9:1 and recrystallization from hexane $/ \mathrm{CH}_{2} \mathrm{Cl}_{2}$ at $0{ }^{\circ} \mathrm{C}$ afforded colorless crystals of both isomers of $\mathbf{2 8}$.

anti,anti-(R,R-Phosphiranyl-1R,3S,7S)- and (S,S-phosphiranyl1S,3R,7R)-isomer 28a: m.p. $150{ }^{\circ} \mathrm{C}$ (decomp); ${ }^{1} \mathrm{H}$ NMR $(400.13 \mathrm{MHz}$, $\left.\mathrm{CDCl}_{3}\right): \delta=0.85-0.98(\mathrm{~m}, 2 \mathrm{H} ; \mathrm{PCH}, \mathrm{PCCH}), 0.99-1.04\left(\mathrm{~m}, 2 \mathrm{H} ; \mathrm{CH}_{2}\right)$, 1.44-1.62 (m, $\left.1 \mathrm{H} ; \mathrm{PCH}_{2} ; \mathrm{m}, 4 \mathrm{H} ; \mathrm{CH}_{2}\right), 1.67\left(\mathrm{ddd},{ }^{2} J(\mathrm{H}, \mathrm{H})=8.7 \mathrm{~Hz}, 1 \mathrm{H}\right.$; $\left.\mathrm{PCH}_{2}\right), 7.38-7.45(\mathrm{~m}, 8 \mathrm{H} ; \mathrm{Ph} H), 7.47-7.53 \mathrm{ppm}(\mathrm{m}, 2 \mathrm{H} ; o-\mathrm{Ph} H) ;{ }^{13} \mathrm{C}\left\{{ }^{1} \mathrm{H}\right\}$ NMR $\left(100.62 \mathrm{MHz}, \mathrm{CDCl}_{3}\right): \delta=8.2\left(\mathrm{~d},{ }^{2} J(\mathrm{C}, \mathrm{P})=3.3 \mathrm{~Hz} ; \mathrm{CH}_{2}\right), 9.7(\mathrm{~s}$; $\left.\mathrm{CH}_{2}\right), 16.0\left(\mathrm{~d},{ }^{1} \mathrm{~J}(\mathrm{C}, \mathrm{P})=11.6 \mathrm{~Hz} ; \mathrm{PCH}_{2}\right), 16.4\left(\mathrm{~s} ; \mathrm{CH}_{2}\right), 24.2\left(\mathrm{~d},{ }^{(2+2} J-\right.$ $(\mathrm{C}, \mathrm{P})=3.5 \mathrm{~Hz} ; \mathrm{PCCH}), 25.1(\mathrm{~s} ; \mathrm{PC}), 27.4\left(\mathrm{~d},{ }^{1} J(\mathrm{C}, \mathrm{P})=16.2 \mathrm{~Hz} ; \mathrm{PCH}\right)$, $31.8\left(\mathrm{dd},{ }^{1} J(\mathrm{C}, \mathrm{P})=3.8,{ }^{3} J(\mathrm{C}, \mathrm{P})=9.6 \mathrm{~Hz} ; \mathrm{PC}\right), 128.6\left(\mathrm{~d},{ }^{3} J(\mathrm{C}, \mathrm{P})=10.5 \mathrm{~Hz}\right.$; $m-\mathrm{Ph}), 129.1\left(\mathrm{~d},{ }^{3} J(\mathrm{C}, \mathrm{P})=10.0 \mathrm{~Hz} ; m-\mathrm{Ph}\right), 130.5\left(\mathrm{~d},{ }^{4} J(\mathrm{C}, \mathrm{P})=2.6 \mathrm{~Hz} ; p-\right.$ $\mathrm{Ph}), 130.8\left(\mathrm{~d},{ }^{4} J(\mathrm{C}, \mathrm{P})=2.1 \mathrm{~Hz} ; p-\mathrm{Ph}\right), 130.9$ (d, ${ }^{1} J(\mathrm{C}, \mathrm{P})=29.8 \mathrm{~Hz}$; ipso$\left.\mathrm{PhPCH}_{2}\right), 132.2\left(\mathrm{~d},{ }^{2} J(\mathrm{C}, \mathrm{P})=13.4 \mathrm{~Hz} ; o-\mathrm{Ph}\right), 132.4\left(\mathrm{~d},{ }^{2} J(\mathrm{C}, \mathrm{P})=12.0 \mathrm{~Hz}\right.$; $o-\mathrm{Ph}), 133.7$ (d, ${ }^{1} J(\mathrm{C}, \mathrm{P})=22.3 \mathrm{~Hz}$; ipso-Ph), $195.3\left(\mathrm{~d},{ }^{2} J(\mathrm{C}, \mathrm{P})=8.3,{ }^{1} J-\right.$ $(\mathrm{C}, \mathrm{W})=125.6 \mathrm{~Hz}$; cis-CO), $195.6\left(\mathrm{~d},{ }^{2} J(\mathrm{C}, \mathrm{P})=8.2,{ }^{1} J(\mathrm{C}, \mathrm{W})=125.7 \mathrm{~Hz}\right.$; cis-CO), $197.2\left(\mathrm{~d},{ }^{2} J(\mathrm{C}, \mathrm{P})=29.9 \mathrm{~Hz}\right.$; trans-CO), $197.8 \mathrm{ppm}\left(\mathrm{d},{ }^{2} J(\mathrm{C}, \mathrm{P})=\right.$ $30.8 \mathrm{~Hz}$; trans-CO); ${ }^{31} \mathrm{P} \mathrm{NMR}\left(101.25 \mathrm{MHz}, \mathrm{CDCl}_{3}\right): \delta=-127.1 \quad\left({ }^{1} J-\right.$ $(\mathrm{P}, \mathrm{W})=258.7 \mathrm{~Hz} ; \quad P[3]$ triangulane $), \quad-164.0 \mathrm{ppm} \quad\left({ }^{1} J(\mathrm{P}, \mathrm{W})=252.6 \mathrm{~Hz}\right.$; PCC); IR (KBr): $\tilde{v}=1919,1936\left(\mathrm{~s} / \mathrm{br}, \mathrm{CO}_{\mathrm{eq}}\right), 1983,1993\left(\mathrm{~s} / \mathrm{br}, \mathrm{CO}_{\mathrm{eq}}\right)$, $2074 \mathrm{~cm}^{-1}\left(\mathrm{~m}, \mathrm{CO}_{\mathrm{ax}}\right)$; elemental analysis calcd $(\%)$ for $\mathrm{C}_{30} \mathrm{H}_{20} \mathrm{O}_{10} \mathrm{P}_{2}{ }^{184} \mathrm{~W}_{2}$ : C 37.14, H 2.08; found: C 36.55, H 2.27.

anti,syn-(R,R-Phosphiranyl-1R,3S,7R)- and (S,S-phosphiranyl-1S,3R,7S)isomer $28 \mathbf{b}$ : m.p. $170{ }^{\circ} \mathrm{C}$; ${ }^{1} \mathrm{H}$ NMR $\left(400.13 \mathrm{MHz}, \mathrm{CDCl}_{3}\right): \delta=0.82-0.89$ $(\mathrm{m}, 1 \mathrm{H} ; \mathrm{PCH}), 1.08-1.12(\mathrm{~m}, 1 \mathrm{H} ; \mathrm{PCCH}), 1.19\left(\mathrm{~m},{ }^{3} J(\mathrm{H}, \mathrm{P})=5.2 \mathrm{~Hz}, 1 \mathrm{H}\right.$; $\left.\mathrm{CH}_{2}\right), 1.21-1.38\left(\mathrm{~m}, 3 \mathrm{H} ; \mathrm{CH}_{2}\right), 1.41-1.54\left(\mathrm{~m}, 2 \mathrm{H} ; \mathrm{CH}_{2}\right), 1.67\left(\mathrm{~m},{ }^{2} \mathrm{~J}-\right.$ $\left.(\mathrm{H}, \mathrm{P})=1.0 \mathrm{~Hz}, 1 \mathrm{H} ; \mathrm{PCH}_{2}\right), 1.97\left(\mathrm{~m},{ }^{2} J(\mathrm{H}, \mathrm{P})=8.4 \mathrm{~Hz}, 1 \mathrm{H} ; \mathrm{PCH}_{2}\right), 7.37-$ $7.41(\mathrm{~m}, 6 \mathrm{H} ; m-\mathrm{Ph} H, p-\mathrm{Ph} H), 7.48-7.54 \mathrm{ppm}(\mathrm{m}, 4 \mathrm{H} ; o-\mathrm{Ph} H) ;{ }^{13} \mathrm{C}\left\{{ }^{1} \mathrm{H}\right\}$ NMR $\left(100.62 \mathrm{MHz}, \mathrm{CDCl}_{3}\right): \delta=8.4\left(\mathrm{~d},{ }^{2} J(\mathrm{C}, \mathrm{P})=3.6 \mathrm{~Hz} ; \mathrm{CH}_{2}\right), 9.9(\mathrm{~s}$; $\left.\mathrm{CH}_{2}\right), 15.2\left(\mathrm{~d},{ }^{2} J(\mathrm{C}, \mathrm{P})=4.6 \mathrm{~Hz} ; \mathrm{CH}_{2}\right), 15.8\left(\mathrm{~d},{ }^{1} \mathrm{~J}(\mathrm{C}, \mathrm{P})=11.4 \mathrm{~Hz} ; \mathrm{PCH}_{2}\right)$, $25.4\left(\mathrm{~d},{ }^{2} J(\mathrm{C}, \mathrm{P})=3.6 \mathrm{~Hz} ; \mathrm{PCCH}\right), 25.9(\mathrm{~s} ; \mathrm{PC}), 27.3\left(\mathrm{dd},{ }^{1} J(\mathrm{C}, \mathrm{P})=15.9,{ }^{3} J-\right.$ $(\mathrm{C}, \mathrm{P})=2.3 \mathrm{~Hz} ; \mathrm{PCH}), 31.4\left(\mathrm{dd},{ }^{1} J(\mathrm{C}, \mathrm{P})=5.3,{ }^{3} J(\mathrm{C}, \mathrm{P})=10.0 \mathrm{~Hz} ; \mathrm{PC}\right)$, $128.6\left(\mathrm{~d},{ }^{3} J(\mathrm{C}, \mathrm{P})=10.7 \mathrm{~Hz} ; m-\mathrm{Ph}\right), 128.9\left(\mathrm{~d},{ }^{3} J(\mathrm{C}, \mathrm{P})=10.1 \mathrm{~Hz} ; m-\mathrm{Ph}\right)$, $130.5\left(\mathrm{~d},{ }^{4} J(\mathrm{C}, \mathrm{P})=2.7 \mathrm{~Hz} ; p-\mathrm{Ph}\right), 130.7\left(\mathrm{~d},{ }^{4} J(\mathrm{C}, \mathrm{P})=2.1 \mathrm{~Hz} ; p-\mathrm{Ph}\right), 130.8$ $\left(\mathrm{d},{ }^{1} \mathrm{~J}(\mathrm{C}, \mathrm{P})=30.0 \mathrm{~Hz}\right.$; ipso- $\left.\mathrm{PhPCH}_{2}\right), 132.5\left(\mathrm{~d},{ }^{2} \mathrm{~J}(\mathrm{C}, \mathrm{P})=13.7 \mathrm{~Hz} ; o-\mathrm{Ph}\right)$, $132.5\left(\mathrm{~d},{ }^{2} J(\mathrm{C}, \mathrm{P})=11.8 \mathrm{~Hz} ; o-\mathrm{Ph}\right), 132.6\left(\mathrm{~d},{ }^{1} J(\mathrm{C}, \mathrm{P})=21.1 \mathrm{~Hz} ;\right.$ ipso- $\left.\mathrm{Ph}\right)$, $195.4\left(\mathrm{~d},{ }^{2} J(\mathrm{C}, \mathrm{P})=8.3,{ }^{1} J(\mathrm{C}, \mathrm{W})=125.5 \mathrm{~Hz}\right.$; cis-CO), 195.7 (d, ${ }^{2} J(\mathrm{C}, \mathrm{P})=$ $8.2,{ }^{1} J(\mathrm{C}, \mathrm{W})=125.7 \mathrm{~Hz}$; cis-CO), 197.1 (d, ${ }^{2} J(\mathrm{C}, \mathrm{P})=29.9 \mathrm{~Hz}$; trans-CO), $197.9 \mathrm{ppm} \quad\left(\mathrm{d},{ }^{2} J(\mathrm{C}, \mathrm{P})=30.6 \mathrm{~Hz} ;\right.$ trans-CO $) ;{ }^{31} \mathrm{P} \mathrm{NMR} \quad(101.25 \mathrm{MHz}$, $\left.\mathrm{CDCl}_{3}\right): \delta=-129.6\left({ }^{1} J(\mathrm{P}, \mathrm{W})=259.7,{ }^{4} J(\mathrm{P}, \mathrm{P})=4.1 \mathrm{~Hz} ; P[3]\right.$ triangulane $)$, $-166.4 \mathrm{ppm}\left({ }^{1} J(\mathrm{P}, \mathrm{W})=251.6,{ }^{4} J(\mathrm{P}, \mathrm{P})=4.1 \mathrm{~Hz} ; \mathrm{PCC}\right)$; IR $(\mathrm{KBr}): \tilde{v}=1898$, 1917 and 1927 (s/br, $\left.\mathrm{CO}_{\mathrm{eq}}\right), 2072 \mathrm{~cm}^{-1}\left(\mathrm{~m}, \mathrm{CO}_{\mathrm{ax}}\right)$; MS (70 eV): $m / z(\%)$ : 970 (20) $[M]^{+}, 914(5)[M-2 \mathrm{CO}]^{+}, 858(12)[M-4 \mathrm{CO}]^{+}, 802 \quad(24)$ $[M-6 \mathrm{CO}]^{+}, 746(42)[M-8 \mathrm{CO}]^{+}, 690(55)[M-10 \mathrm{CO}]^{+}$; HR-MS (EI): $m / z$ : calcd for $\mathrm{C}_{30} \mathrm{H}_{20} \mathrm{O}_{10} \mathrm{P}_{2}{ }^{184} \mathrm{~W}_{2}$ : 969.95520 ; found: 969.95191 .

Crystal structure determinations: $\mathrm{X}$-ray intensities were measured on a Nonius KappaCCD diffractometer with rotating anode and graphite monochromator $(\lambda=0.71073 \AA)$. The structures were solved with automated Patterson Methods ${ }^{[34]}$ (compounds 7, 12a, 14, 16b, 21 a, and 28a) or Direct Methods ${ }^{[35]}$ (compound 17a) and refined with SHELXL-97 $7^{[36]}$ on $F^{2}$ of all reflections. Geometry calculations, drawings and checking for higher symmetry were performed with the PLATON ${ }^{[37]}$ package.

Compound 7: $\mathrm{C}_{25} \mathrm{H}_{21} \mathrm{O}_{5} \mathrm{PW}, F_{\mathrm{w}}=616.24$, colourless needle, $0.51 \times 0.24 \times$ $0.21 \mathrm{~mm}^{3}$, monoclinic, $P 2_{1} / c$ (no. 14), $a=8.0553(1), b=17.0487(2), c=$ 18.6096(3) ̊, $\quad \beta=114.6694(12)^{\circ}, \quad V=2322.45(6) \AA^{3}, \quad Z=4, \quad \rho=$ $1.762 \mathrm{~g} \mathrm{~cm}^{-3} ; T=110(2) \mathrm{K} ; 54513$ reflections were measured up to a resolution of $(\sin \theta / \lambda)_{\max }=0.81 \AA^{-1}$. An analytical absorption correction was applied $\left(\mu=5.08 \mathrm{~mm}^{-1}, 0.15-0.40\right.$ correction range). 10119 reflections were unique $\left(R_{\text {int }}=0.049\right)$. Non-hydrogen atoms were refined freely with anisotropic displacement parameters. All hydrogen atoms were located in the difference Fourier map. Phenyl hydrogen atoms were refined as rigid groups, all other hydrogen atoms were refined freely with isotropic displacement parameters. 353 parameters were refined with no restraints. $R 1 / w R 2$ [ $I>2 \sigma(I)$ ]: $0.0188 / 0.0416 . R 1 / w R 2$ [all refl.]: $0.0412 / 0.0533 . S=$ 0.603 . Residual electron density between -1.32 and $1.63 \mathrm{e}^{-3}$.

Compound 12a: $\mathrm{C}_{17} \mathrm{H}_{13} \mathrm{O}_{5} \mathrm{PW}, F_{\mathrm{w}}=512.09$, colourless plate, $0.18 \times 0.13 \times$ $0.06 \mathrm{~mm}^{3}$, monoclinic, $P 2_{1} / c$ (no. 14), $a=9.8460(1), b=9.2242(1), c=$ 38.9935(3) ̊, $\beta=97.1897(3)^{\circ}, V=3513.60(6) \AA^{3}, Z=8, \rho=1.936 \mathrm{~g} \mathrm{~cm}^{-3}$; $T=150(2) \mathrm{K} ; 44020$ reflections were measured up to a resolution of $(\sin \theta / \lambda)_{\max }=0.65 \AA^{-1}$. An analytical absorption correction was applied ( $\mu=6.69 \mathrm{~mm}^{-1}, 0.32-0.79$ correction range). 7989 reflections were unique $\left(R_{\text {int }}=0.041\right)$. Non-hydrogen atoms were refined freely with anisotropic displacement parameters. All hydrogen atoms were located in the difference Fourier map. Phenyl hydrogen atoms were refined as rigid groups, all other hydrogen atoms were refined freely with isotropic displacement parameters. 497 parameters were refined with no restraints. $R 1 / w R 2[I>$ $2 \sigma(I)$ ]: $0.0211 / 0.0432 . R 1 / w R 2$ [all refl.]: $0.0310 / 0.0461 . S=1.026$. Residual

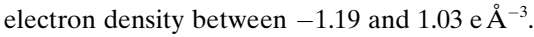

Compound 14: $\mathrm{C}_{19} \mathrm{H}_{15} \mathrm{O}_{5} \mathrm{PW}, F_{\mathrm{w}}=538.13$, colourless block, $0.33 \times 0.15 \times$ $0.15 \mathrm{~mm}^{3}$, monoclinic, $C 2 / c$ (no. 15), $a=24.8645(2), b=10.0503(1), c=$ $18.0840 \AA ., \beta=122.9428(4)^{\circ}, V=3792.50(7) \AA^{3}, \quad Z=8, \rho=1.865 \mathrm{~g} \mathrm{~cm}^{-3}$; $T=150(2) \mathrm{K} ; 32020$ reflections were measured up to a resolution of $(\sin \theta / \lambda)_{\max }=0.65 \AA^{-1}$. An analytical absorption correction was applied $\left(\mu=6.20 \mathrm{~mm}^{-1}, 0.21-0.43\right.$ correction range). 4335 reflections were unique $\left(R_{\text {int }}=0.039\right)$. Non-hydrogen atoms were refined freely with anisotropic displacement parameters. All hydrogen atoms were located in the difference Fourier map. Phenyl hydrogen atoms were refined as rigid groups, all other hydrogen atoms were refined freely with isotropic displacement parameters. 275 parameters were refined with no restraints. $R 1 / w R 2[I>$ $2 \sigma(I)$ ]: $0.0165 / 0.0365 . R 1 / w R 2$ [all refl.]: $0.0192 / 0.0374 . S=1.097$. Residual

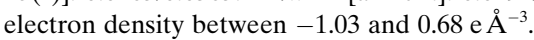

Compound 16b: $\mathrm{C}_{18} \mathrm{H}_{13} \mathrm{O}_{5} \mathrm{PW}, F_{\mathrm{w}}=524.10$, colourless needle, $0.36 \times 0.09 \times$ $0.06 \mathrm{~mm}^{3}$, triclinic, $P \overline{1}$ (no. 2), $a=9.9006(10), \quad b=14.0474(12), \quad c=$ 14.652(2) А̊, $\alpha=64.984(8), \beta=79.165(9), \gamma=89.522(8)^{\circ}, V=1807.9(3) \AA^{3}$, $Z=4, \rho=1.926 \mathrm{~g} \mathrm{~cm}^{-3} ; T=150(2) \mathrm{K} ; 41602$ reflections were measured up to a resolution of $(\sin \theta / \lambda)_{\max }=0.59 \AA^{-1}$. An analytical absorption correction was applied $\left(\mu=6.50 \mathrm{~mm}^{-1}, 0.24-0.86\right.$ correction range). The crystal appeared to be non-merohedrally twinned with a twofold rotation about the a-axis as twin operation. This twin relationship was taken into account for the integration ${ }^{[38]}$ and merging ${ }^{[39]}$ of the reflections and the HKLF5 refinement. ${ }^{[40]} 11455$ reflections were unique $\left(R_{\text {int }}=0.071\right)$. Nonhydrogen atoms were refined freely with anisotropic displacement parameters. All hydrogen atoms were located in the difference Fourier map 
and refined as rigid groups. 452 parameters were refined with no restraints. $R 1 / w R 2[I>2 \sigma(I)]: 0.0422 / 0.1208$. $R 1 / w R 2$ [all refl.]: $0.0746 /$ 0.1343 . $\mathrm{S}=1.084$. Twin fraction $0.1862(5)$. Residual electron density between -1.36 and $2.04 \mathrm{e}^{-3}$.

Compound 17a: $\mathrm{C}_{29} \mathrm{H}_{18} \mathrm{O}_{10} \mathrm{P}_{2} \mathrm{~W}_{2}, F_{\mathrm{w}}=956.07$, colourless needle, $0.42 \times$ $0.12 \times 0.12 \mathrm{~mm}^{3}$, monoclinic, $P 2_{1}$ (no. 4), $a=6.3432(4), b=21.233(2), c=$ 11.6997(8) ̊, $\beta=98.997(7)^{\circ}, V=1556.4(2) \AA^{3}, Z=2, \rho=2.040 \mathrm{~g} \mathrm{~cm}^{-3} ; T=$ $150(2)$ K. 18806 reflections were measured up to a resolution of $(\sin \theta /$ $\lambda)_{\max }=0.65 \AA^{-1}$. An absorption correction based on multiple measured reflections was applied $\left(\mu=7.54 \mathrm{~mm}^{-1}, 0.18-0.41\right.$ correction range) 7049 reflections were unique $\left(R_{\text {int }}=0.026\right)$. Non-hydrogen atoms were refined freely with anisotropic displacement parameters. Hydrogen atoms were introduced in calculated positions and refined as rigid groups. 388 parameters were refined with 1 restraint. $R 1 / w R 2[I>2 \sigma(I)]: 0.0296 / 0.0667$. $R 1 / w R 2$ [all refl.]: $0.0359 / 0.0699 . \quad S=1.018$. Flack $x$ parameter ${ }^{[41]}$ $-0.024(8)$. Residual electron density between -1.79 and $1.26 \mathrm{e} \AA^{-3}$.

Compound 21 a: $\mathrm{C}_{19} \mathrm{H}_{15} \mathrm{O}_{5} \mathrm{PW}, F_{\mathrm{w}}=538.13$, colourless needle, $0.20 \times 0.08 \times$ $0.04 \mathrm{~mm}^{3}, \quad$ triclinic, $P \overline{1} \quad($ no. 2$), \quad a=9.1520(1), \quad b=9.2457(1), \quad c=$ 11.7216(2) ̊ $, \quad \alpha=79.5224(9), \quad \beta=72.9620(8), \quad \gamma=84.7207(7)^{\circ}, \quad V=$ 931.71(2) $\AA^{3}, Z=2, \rho=1.918 \mathrm{~g} \mathrm{~cm}^{-3} ; T=150$ (2) $\mathrm{K} ; 16007$ reflections were measured up to a resolution of $(\sin \theta / \lambda)_{\max }=0.65 \AA^{-1}$. An analytical absorption correction was applied $\left(\mu=6.31 \mathrm{~mm}^{-1}, 0.39-0.77\right.$ correction range). 4172 reflections were unique $\left(R_{\text {int }}=0.045\right)$. Non-hydrogen atoms were refined freely with anisotropic displacement parameters. All hydrogen atoms were located in the difference Fourier map. Phenyl hydrogen atoms were refined as rigid groups, all other hydrogen atoms were refined freely with isotropic displacement parameters. 275 parameters were refined with no restraints. $R 1 / w R 2[I>2 \sigma(I)]: 0.0192 / 0.0386 . R 1 / w R 2$ [all refl.]: $0.0236 / 0.0397$. $S=1.054$. Residual electron density between -0.84 and $0.98 \mathrm{e}^{-3}$.

Compound 28a: $\mathrm{C}_{30} \mathrm{H}_{20} \mathrm{O}_{10} \mathrm{P}_{2} \mathrm{~W}_{2}+$ disordered solvent, $F_{\mathrm{w}}=970.10[*]$, yellowish needle, $0.42 \times 0.18 \times 0.18 \mathrm{~mm}^{3}$, monoclinic, $C 2 / c$ (no. 15), $a=$ $30.9063(16), \quad b=11.9175(8), \quad c=20.6181(11) \AA, \quad \beta=117.985(7)^{\circ}, \quad V=$ 6706.2(8) $\AA^{3}, Z=8, \rho=1.922 \mathrm{~g} \mathrm{~cm}^{-3}[*] ; T=150(2) \mathrm{K} ; 49557$ reflections were measured up to a resolution of $(\sin \theta / \lambda)_{\max }=0.65 \AA^{-1}$. An absorption correction based on multiple measured reflections was applied $(\mu=$ $7.00 \mathrm{~mm}^{-1}[*], 0.17-0.28$ correction range). Non-hydrogen atoms were refined freely with anisotropic displacement parameters. All hydrogen atoms were located in the difference Fourier map. Phenyl hydrogen atoms were refined as rigid groups, all other hydrogen atoms were refined freely with isotropic displacement parameters. The crystal structure contains large voids ( $545 \AA^{3} /$ unit cell) filled with disordered $\mathrm{CH}_{2} \mathrm{Cl}_{2}$ solvent molecules. Their contribution to the structure factors was secured by back-Fourier transformation using the SQUEEZE routine of the PLATON program ${ }^{[37]}$ resulting in 152 electrons/unit cell. 437 parameters were refined with no restraints. $R 1 / w R 2[I>2 \sigma(I)]: 0.0190 / 0.0370 . R 1 /$ $w R 2$ [all refl.]: $0.0297 / 0.0386 . S=1.030$. Residual electron density between -0.64 and $0.76 \mathrm{e}^{-3}$. [*] Derived quantities do not contain the contribution of the disordered solvent

CCDC-212086 (7), -269693 (12a), -269694 (14), -269695 (16b), -269696 (17a), -269697 (21a) and -269698 (28a) contain the supplementary crystallographic data for this paper. These data can be obtained free of charge from The Cambridge Crystallographic Data Centre via www.ccdc.cam.ac.uk/data_request/cif/.

\section{Acknowledgements}

This work was supported by the Council for Chemical Sciences of the Netherlands Organization for Scientific Research (CW/NWO). Dr. H. Zappey is thanked for the HR-MS measurements. Work of the German group was supported by the State of Niedersachsen and the Fonds der Chemischen Industrie.

[1] a) Cyclopropanes and Related Rings (Ed.: A. de Meijere), Chem. Rev. 2003, 103, 931-1625 whole Issue; b) Carbocyclic Three-Mem- bered Ring Compounds, in Methods of Organic Chemistry (HoubenWeyl), Vol. E17a-c (Ed.: A. de Meijere), Thieme, Stuttgart, 1997; c) The Chemistry of the Cyclopropyl Group, Vol. 2 (Ed.: Z. Rappoport), Wiley, Chichester, 1995; d) A. de Meijere, Angew. Chem. 1979, 91, 867-884; Angew. Chem. Int. Ed. Engl. 1979, 18, 809-826.

[2] A. de Meijere, S. I. Kozhushkov, Chem. Rev. 2000, 100, 93-142.

[3] a) M. von Seebach, S. I. Kozhushkov, R. Boese, J. Benet-Buchholz, D. S. Yufit, J. A. K. Howard, A. de Meijere, Angew. Chem. 2000, 112, 2617-2620; Angew. Chem. Int. Ed. 2000, 39, 2495-2498; b) A. de Meijere, M. von Seebach, S. Zöllner, S. I. Kozhushkov, V. N. Belov, R. Boese, T. Haumann, J. Benet-Buchholz, D. S. Yufit, J. A. K. Howard, Chem. Eur. J. 2001, 7, 4021-4034; c) A. de Meijere, A. F. Khlebnikov, S. I. Kozhushkov, K. Miyazawa, D. Frank, P. R. Schreiner, C. Rinderspacher, D. S. Yufit, J. A. K. Howard, Angew. Chem. 2004, 116, 6715-6719; Angew. Chem. Int. Ed. 2004, 43, 6553-6557.

[4] J. -T. Hung, S.-W. Yang, G. M. Gray, K. Lammertsma, J. Org. Chem. 1993, 58, 6786-6790.

[5] K. Lammertsma, B. Wang, J. -T. Hung, A. W. Ehlers, G. M. Gray, J. Am. Chem. Soc. 1999, 121, 11650-11655.

[6] M. J. M. Vlaar, M. H. Lor, A. W. Ehlers, M. Schakel, M. Lutz, A. L. Spek, K. Lammertsma, J. Org. Chem. 2002, 67, 2485-2493.

[7] N. H. Tran Huy, R. Salemkour, N. Bartes, L. Ricard, F. Mathey, Tetrahedron 2002, 58, 7191-7193.

[8] a) F. Mathey, Chem. Rev. 1990, 90, 997-1025; b) F. Mathey, M. Regitz, In Phosphorus-Carbon Heterocyclic Chemistry: The Rise of a New Domain (Ed.: F. Mathey), Pergamon, Amsterdam, 2001, pp. $17-55$.

[9] A. Marinetti, F. Mathey, J. Fischer, A. Mitschler, J. Am. Chem. Soc. 1982, 104, 4484-4485.

[10] a) K. Lammertsma, M. J. M. Vlaar, Eur. J. Org. Chem. 2002, 1127 1138; b) F. Mathey, N. H. Tran Huy, A. Marinetti, Helv. Chim. Acta 2001, 84, 2938-2957.

[11] J. C. Slootweg, M. Schakel, F. J. J. de Kanter, A. W. Ehlers, S. I. Kozhushkov, A. de Meijere, M. Lutz, A. L. Spek, K. Lammertsma, J. Am. Chem. Soc. 2004, 126, 3050-3051.

[12] A. Marinetti, F. Mathey, Organometallics 1984, 3, 456-461.

[13] A. Marinetti, F. Mathey, Organometallics 1982, 1, 1488-1492.

[14] a) R. Boese, T. Miebach, A. de Meijere, J. Am. Chem. Soc. 1991, 113, 1743-1748; b) S. Zöllner, H. Buchholz, R. Boese, R. Gleiter, A. de Meijere, Angew. Chem. 1991, 103, 1544-1546; Angew. Chem. Int. Ed. Engl. 1991, 30, 1518-1520; c) R. Boese, T. Haumann, E. D. Jemmis, B. Kiran, S. Kozhushkov, A. de Meijere, Liebigs Ann. 1996, 913-919.

[15] J. -T. Hung, S. -W. Yang, P. Chand, G. M. Gray, K. Lammertsma, J. Am. Chem. Soc. 1994, 116, 10966-10971.

[16] B. Wang, C. H. Lake, K. Lammertsma, J. Am. Chem. Soc. 1996, 118, $1690-1695$

[17] P. J. Stang, J. R. Madsen, M. G. Mangum, D. P. Fox, J. Org. Chem. 1977, 42, 1802-1804.

[18] A. Marinetti, C. Charrier, F. Mathey, J. Fischer, Organometallics 1985, 4, 2134-2138.

[19] M. J. van Eis, F. J. J. de Kanter, W. H. de Wolf, K. Lammertsma, F. Bickelhaupt, M. Lutz, A. L. Spek, Tetrahedron 2000, 56, 129-136.

[20] K. Lammertsma, A. W. Ehlers, M. L. McKee, J. Am. Chem. Soc. 2003, 125, 14750-14759.

[21] a) A. de Meijere, S. I. Kozhushkov, A. F. Khlebnikov, Top. Curr Chem. 2000, 207, 89-147; b) A. de Meijere, S. I. Kozhushkov, Eur. J. Org. Chem. 2000, 3809-3822.

[22] a) M. J. M. Vlaar, F. J. J. de Kanter, M. Schakel, M. Lutz, A. L. Spek, K. Lammertsma, J. Organomet. Chem. 2001, 617-618, 311-317; b) B. Deschamps, F. Mathey, J. Chem. Soc. Chem. Commun. 1985, 1010-1012; c) B. Deschamps, F. Mathey, J. Organomet. Chem. 1988, $354,83-90$.

[23] R. E. Bulo, A. W. Ehlers, F. J. J. de Kanter, M. Schakel, M. Lutz, A. L. Spek, K. Lammertsma, B. Wang, Chem. Eur. J. 2004, 10, $2732-$ 2738.

[24] For reviews see: a) A. J. H. Klunder, B. Zwanenburg In Methods of Organic Chemistry (Houben-Weyl), Vol. E17c (Ed.: A. de Meijere), 
Thieme, Stuttgart, 1997, pp. 2419-2537; b) L. Fitjer in Methods of Organic Chemistry (Houben-Weyl), Vol. E17c (Ed.: A. de Meijere), Thieme, Stuttgart, 1997, pp. 251-317.

[25] S. Kozhushkov, T. Späth, T. Fiebig, B. Galland, M. -F. Ruasse, P. Xavier, Y. Apeloig, A. de Meijere, J. Org. Chem. 2002, 67, 41004114.

[26] All attempts to synthesize an oxa[7]triangulane by epoxidation of the second-generation bicyclopropylidene $\mathbf{1 8}$ failed and an oligospirocyclopropanated cyclobutanone was obtained: D. Frank, S. I. Kozhushkov, T. Labahn, A. de Meijere, Tetrahedron 2002, 58, $7001-$ 7007.

[27] R. E. Bulo, A. W. Ehlers, S. Grimme, K. Lammertsma, J. Am. Chem. Soc. 2002, 124, 13903-13910. This rearrangement of $\mathbf{3 0}$ is a heteroanalogue of the vinylcyclopropane-cyclopentene rearrangement: J.E. Baldwin, in The Chemistry of the Cyclopropyl, Vol. 2 (Ed.: Z. Rappoport), Wiley, Chichester, 1995, pp. 469-494; J. E. Baldwin, J. Comput. Chem. 1998, 19, 222-231; J. E. Baldwin, Chem. Rev. 2003, 103, 1197-1212.

[28] S. Arora, P. Binger, Synthesis 1974, 801-803.

[29] a) N. S. Zefirov, K. A. Lukin, S. I. Kozhushkov, T. S. Kuznetsova, A. M. Domarev, I. M. Sosonkin, Zh. Org. Khim. 1989, 25, 312-319; J. Org. Chem. USSR (Engl. Transl.) 1989, 25, 278-284; b) A. de Meijere, A. F. Khlebnikov, S. I. Kozhushkov, R. R. Kostikov, P. R. Schreiner, A. Wittkopp, C. Rinderspacher, D. S. Yufit, J. A. K. Howard, Chem. Eur. J. 2002, 8, 828-842.

[30] I. Erden, Synth. Commun. 1986, 16, 117-121.
[31] K. A. Lukin, A. Y. Masunova, B. I. Ugrak, N. S. Zefirov, Tetrahedron 1991, 47, 5769-5780.

[32] A. de Meijere, S. I. Kozhushkov, T. Spaeth, N. S. Zefirov, J. Org. Chem. 1993, 58, 502-505.

[33] A. de Meijere, S. I. Kozhuskov, D. Faber, V. Bagutskii, R. Boese, T. Haumann, R. Walsh, Eur. J. Org. Chem. 2001, 3607-3614.

[34] P. T. Beurskens, G. Admiraal, G. Beurskens, W. P. Bosman, S. Garcia-Granda, R. O. Gould, J. M.M. Smits, C. Smykalla, The DIRDIF99 program system, Technical Report of the Crystallography Laboratory, University of Nijmegen, The Netherlands, 1999.

[35] A. Altomare, M. C. Burla, M. Camalli, G. L. Cascarano, C. Giacovazzo, A. Guagliardi, A. G. G. Moliterni, G. Polidori, R. Spagna, $J$. Appl. Crystallogr. 1999, 32, 115-119.

[36] G. M. Sheldrick, SHELXL-97. Program for crystal structure refinement, Universität Göttingen (Germany), 1997.

[37] A. L. Spek, J. Appl. Crystallogr. 2003, 36, 7-13.

[38] A. J. M. Duisenberg, L. M. J. Kroon-Batenburg, A. M. M. Schreurs, J. Appl. Crystallogr. 2003, 36, 220-229.

[39] A. M. M. Schreurs, MERGEHKLF5, Utrecht University (The Netherlands), 2005

[40] R. Herbst-Irmer, G. S. Sheldrick, Acta Crystallogr. Sect. B 1998, 54, 443-449.

[41] H. D. Flack, Acta Crystallogr. Sect. A 1983, 39, 876-881.

Received: May 13, 2005 Published online: August 3, 2005 